\title{
Biosynthetic interrelationships within polycyclic cembranoids isolated from corals. Conjecture, biomimetic synthesis and reality.
}

\author{
Matthew J. Palframan ${ }^{[a]}$ and Gerald Pattenden ${ }^{\star[a]}$
}

\begin{abstract}
Macrocyclic furanobutenolide-based cembranoids are precursors to a wide variety of complex ring-fused diterpene structures in corals, implicating a wide variety of oxidation and photochemical processes, cyclisation and transannulation reactions, and skeletal rearrangements from a variety of reactive intermediates and pericyclic processes. This article gives an up to date personal perspective on the speculations that underpin these interesting biosynthetic interrelationships, and summarises biomimetic synthesis and interconversions that would seem to vindicate some of these speculations.
\end{abstract}

\section{Table of Contents}

1 Introduction and scope

2 Bielschowskysin

3 Plumarellide and rameswaralide

4 Intricarene

5 Ineleganolide and related norcembranoids

6 Other cembranoid-derived secondary metabolites

7 Concluding remarks

8 References and notes

\section{Introduction and scope ${ }^{\dagger}$}

Furanobutenolide-based cembranoids (FBCs) are a large family of 14-membered ring diterpenes derived from gerany geranyl pyrophosphate which accommodate furan rings between C3-C6 and butenolide ring systems encompassing $\mathrm{C} 10-\mathrm{C} 12$ in their
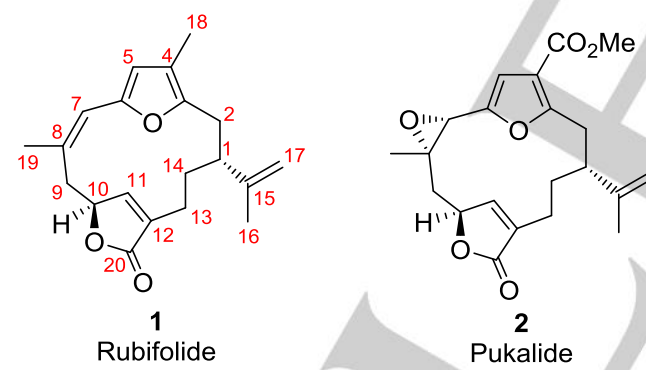

structures. ${ }^{[1-4]}$ They have been isolated exclusively from corals and all are related to the core rubifolide structure 1 . The first of their number to be characterised however was pukalide 2, from the soft coral Sinularia abrupta by Scheuer et al. in 1975 . Other representative members are bipinnatin $\mathrm{J}(3)$, and the muscular neurotoxin lophotoxin 4 (Fig.1) The many oxy-substituted FBCs in Nature are either products of biological epoxidations at $\mathrm{C} 7-\mathrm{C} 8$ and $\mathrm{C} 11-\mathrm{C} 12$ ( also $\mathrm{C} 15-\mathrm{C} 17$ ) or oxidations at $\mathrm{C} 13, \mathrm{C} 14, \mathrm{C} 16$ and $\mathrm{C} 18$, or both, in the core structure 1 . Although most FBCs have Z-configurations at their C7-C8 alkene bonds a few, like acerosolide 5, show the alternative E-configuration at the same alkene bond. ${ }^{[1]}$ Enedione-based cembranoids, e.g. coralloidolide $E$ (6) resulting from oxidative cleavage of the furan rings in $\mathrm{FBCs},{ }^{[5]}$ and isomeric structures with 12-membered rings known as pseudopterolides or kallolides, ${ }^{[6]}$ e.g. kallolide $\mathrm{B}(7)$ are also commonly found alongside FBCs in corals.

By far the most exciting FBC-related metabolites to be found in corals however are a variety of highly oxygenated and complex

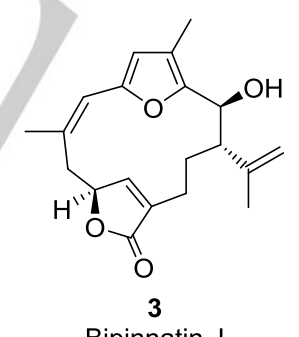

Bipinnatin J

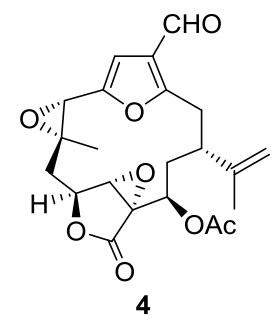

Lophotoxin
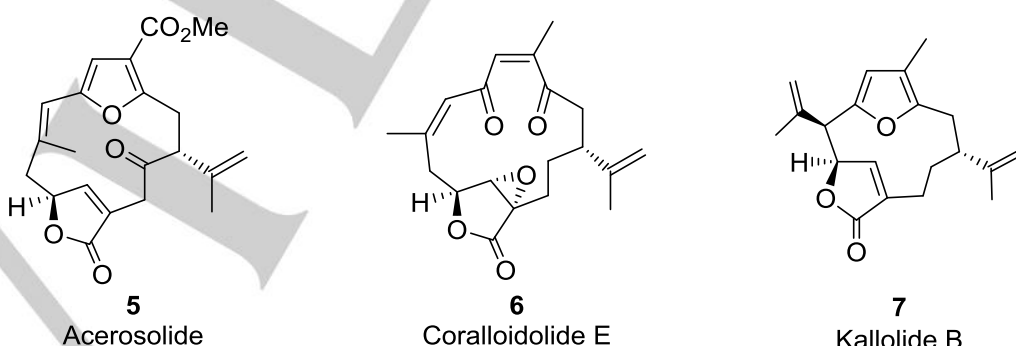

Figure 1. Some furanobutenolide-based cembranoids, (FBCs), and oxidised relatives isolated from corals.

[a] School of Chemistry, The University of Nottingham, University Park, Nottingham, NG7 2RD, UK

E-mail: GP@nottingham.ac.uk Homepage: https://www.nottingham.ac.uk/ pczgp1/pattenden$\underline{\text { main.html }}$

\footnotetext{
${ }^{\dagger}$ Footnote: Gerry Pattenden lectured at the Bristol Synthesis Meeting during 2008, and on that occasion he presented the total synthesis of three widely different natural products, i.e. ulapualide A, salinosporamide $A$ and intricarene. Over the ensuing recent years his research group has investigated biomimetic synthetic approaches to a range of complex polycyclic natural diterpenoids found in corals. This personal Perspective summarises the progress his group has made in this area, alongside contemporaneous studies of others.
} 


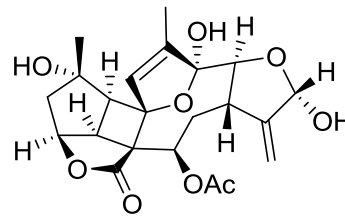

8 Bielschowskysin

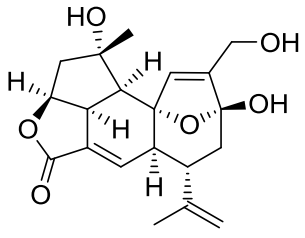

9

Plumarellide

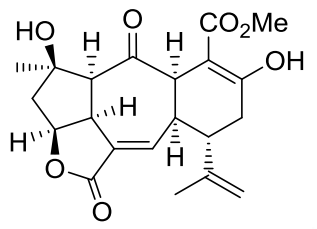

10

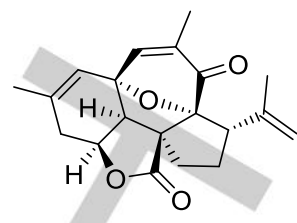

11

Rameswaralide

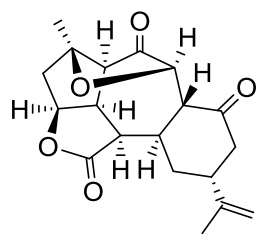

12 Ineleganolide

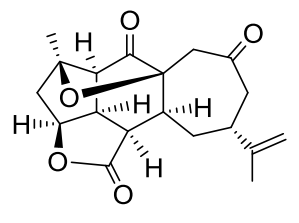

13

Sinulochmodin C

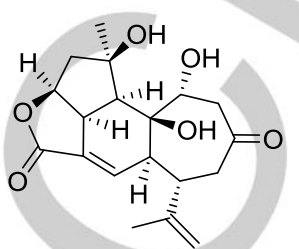

14

Dissectolide A

Figure 2. Examples of polycyclic cembranoids and related norcembranoids isolated from corals.

ring-fused diterpene metabolites, represented by bielschowskysin $8,{ }^{[7]}$ plumarellide $9,{ }^{[8]}$ rameswaralide $10,{ }^{[9]}$ and intricarene 11. ${ }^{[10]}$ In addition, a number of related $\mathrm{C} 19$ norditerpenes, which also have highly complex ring-fused systems, e.g. ineleganolide $12{ }^{[11]}$ sinulochromodin $C(13)^{[12]}$ and dissectolide $14,{ }^{[13]}$ have been isolated from corals and clearly have their origins in FBC precursors. The aristocratic nature, combined with the unusual fusion of rings with complex oxysubstitution patterns and stereochemistry in the polycyclic structures 8-14 have enticed and seduced a number of synthetic chemists to take up the challenge of their synthesis in the lab. A recent review has summarised a variety of synthetic approaches that have been made towards members of the polycyclic structures 8-14, especially towards bielschowsksin 8 and ineleganolide 12. ${ }^{[14]}$ In this Perspective we focus on speculations that have been made, by us and others, over the past decade on the most likely biosynthesis pathways to the aforementioned polycycles and some of their relatives, and then summarise the progress that has been made to vindicate some of these speculations in the lab., i.e. by biomimetic syntheses.

In needs to be mentioned that the marine milieu is not the ideal environment to conduct studies of the biosynthesis of polycyclic structures like 8-14 and indeed few have been published. Nevertheless, the marine milieu does have oxygen and water, and contains metals and salts for triggering a variety of oxidations, hydrolyses and $\mathrm{C} \rightarrow \mathrm{C}$ bond forming reactions and, of course' there is sunlight for catalysing an array of novel photochemical rearrangements and other bond-forming reactions. All of these "opportunities" for the possible elaboration of the polycyclic structures 8-14 and their relatives from macrocyclic FBC starting materials will be discussed and evaluated in this Perspective.

Earlier, in 2011, we presented our speculations on some of these events in two reviews published in Natural Product Reports. Since that time several new polycyclic cembranoid secondary metabolites have been found in corals, and more
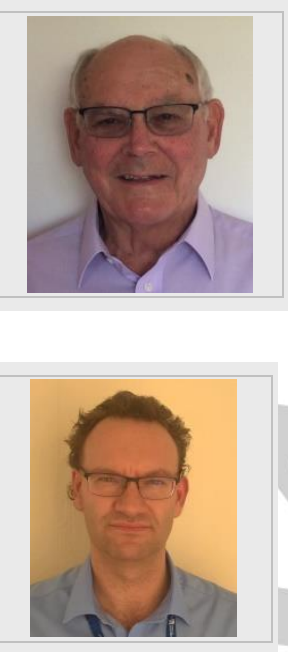

Gerry Pattenden is Emeritus Professor in the School of Chemistry at The University of Nottingham. He was Sir Jesse Boot Professor of Organic Chemistry until 2005 and then Research Professor until his supposed retirement in 2009. However, Gerry has continued to do research and publish, and his most recent publications (with Matthew Palframan) describe the structure and chemistry of a new and exciting insect pheromone, named sobralene, isolated from males of the Brazilian sand fly. Sobralene has a close biosynthetic relationship with its co-metabolite taxadiene which he is now studying further. Over his career Gerry's research has been driven largely by his curiosity of biosynthetic interrelationships within several families of natural products. Alongside studies of these relationships, largely through synthesis, he also discovered and developed a wide range of cascade radical reactions leading to steroids and polycyclic terpenes, and synthesised a wide variety of natural products including complex polyene and azole-based macrocyclic compounds.

Matthew Palframan graduated from Oxford University with an MChem, and then carried out research with Professor Andy Parsons at the University of York on the synthesis of alkaloids leading to a PhD in 2010. After post-doctoral research exploiting the products of microbial oxidations in synthesis with Dr Simon Lewis at the University of Bath, Matthew joined the research group of Professor Pattenden in Nottingham where he explored biomimetic intramolecular cycloaddition reactions for the construction of complex ring systems found in diterpenes, such a rameswaralide and plumarellide isolated from corals. Subsequently Matthew conducted research as a medicinal chemist in the labs of Prof. Chris Hayes in Nottingham. He is now a Lecturer in Medicinal Chemistry in the School of Pharmacy at The University of Wolverhampton. 
biosynthesis speculations as to their possible origins have been forthcoming. To provide a coherent survey and Perspective on this recent progress it has been necessary to summarise some of the ideas we have reviewed previously. We apologies for this and hope that we have not missed any important earlier work or, indeed offended any colleagues who may feel that some of their studies have not been considered in sufficient detail or even overlooked.

\section{Bielschowsyksin}

Bielschowskysin $\mathbf{8}$ accommodates a rare substitutedcyclobutane ring embedded within its unique and complex polycyclic structure. It is also reported to exhibit cytotoxicity against non-small lung cancer cells and renal cancer cells. Its challenging structure and interesting biological properties have combined to make bielschowskysin one of the most revered targets amongst synthetic chemists in recent years. ${ }^{[14]}$

The complex fused ring system in bielschowskysin $\mathbf{8}$ is thought to originate in vivo from the FBC structure bipinnatin $\mathrm{J}(3)$ after a sequence of regio- and stereo-selective oxidations, which first lead to the epoxide 15 , followed by acid-catalysed hydration producing the exocyclic enol ether cyclic hemiketal 16 as key intermediate (Scheme 1)..$^{[1,2]}$ Intramolecular [2+2] photocycloaddition involving the butenolide and enol ether alkene bonds in $\mathbf{1 6}$ would then lead to the natural product. The enol ether cyclic hemiketal unit in $\mathbf{1 6}$ is the tautomer of the cyclic enedione 17 and an alternative ionic pathway to bielschowskysin from the enolate produced from 17, involving sequential Michael and aldol reactions between $\mathrm{C} 7$ and $\mathrm{C} 11$, and between $\mathrm{C} 12$ and C6 in 17, viz, $\mathbf{1 7 \rightarrow 1 8} \rightarrow \mathbf{1 9}$ (Scheme 2), has also been suggested. ${ }^{[1]}$
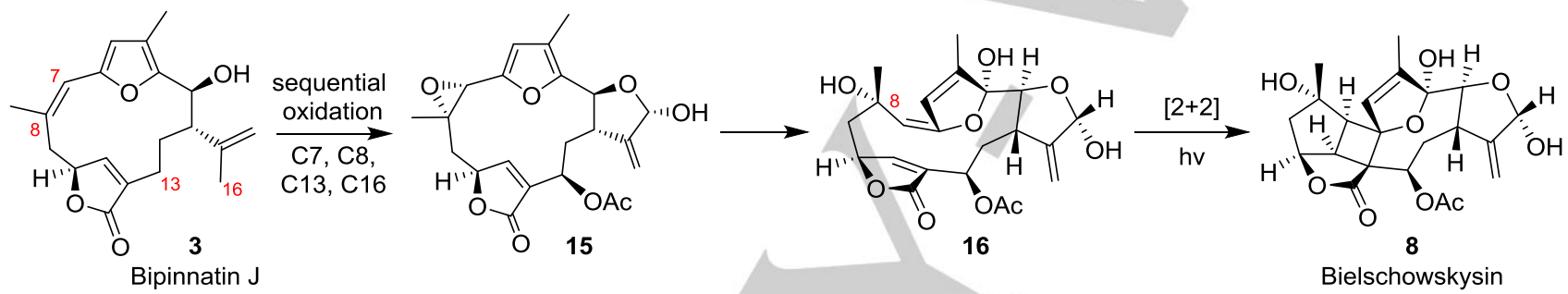

Scheme 1 Proposal for the biosynthesis of bielschowskysin 8 from bipinatin $\mathrm{J}(3)$ featuring an intramolecular [2+2] photocycloaddition in the enol ether cyclic hemiketal intermediate 16

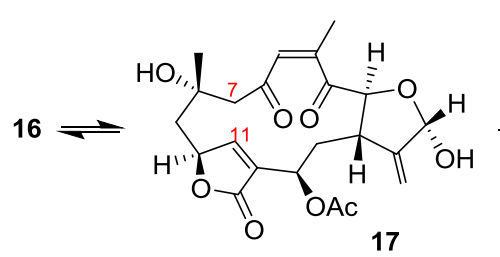

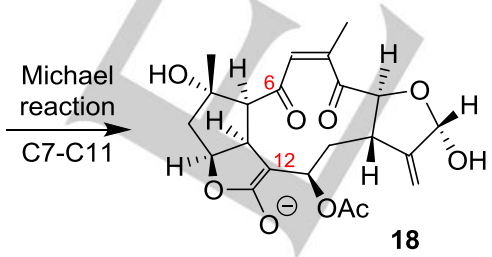
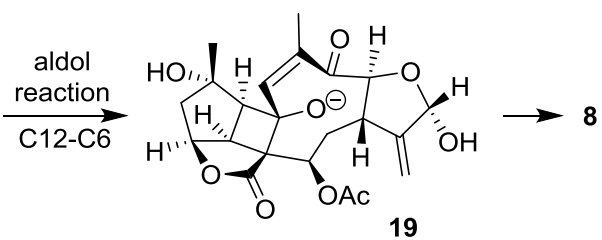

Scheme 2. An alternative proposal for the biosynthesis of bielschowskysin 8 from the enedione intermediate 17 via anionic intermediates.

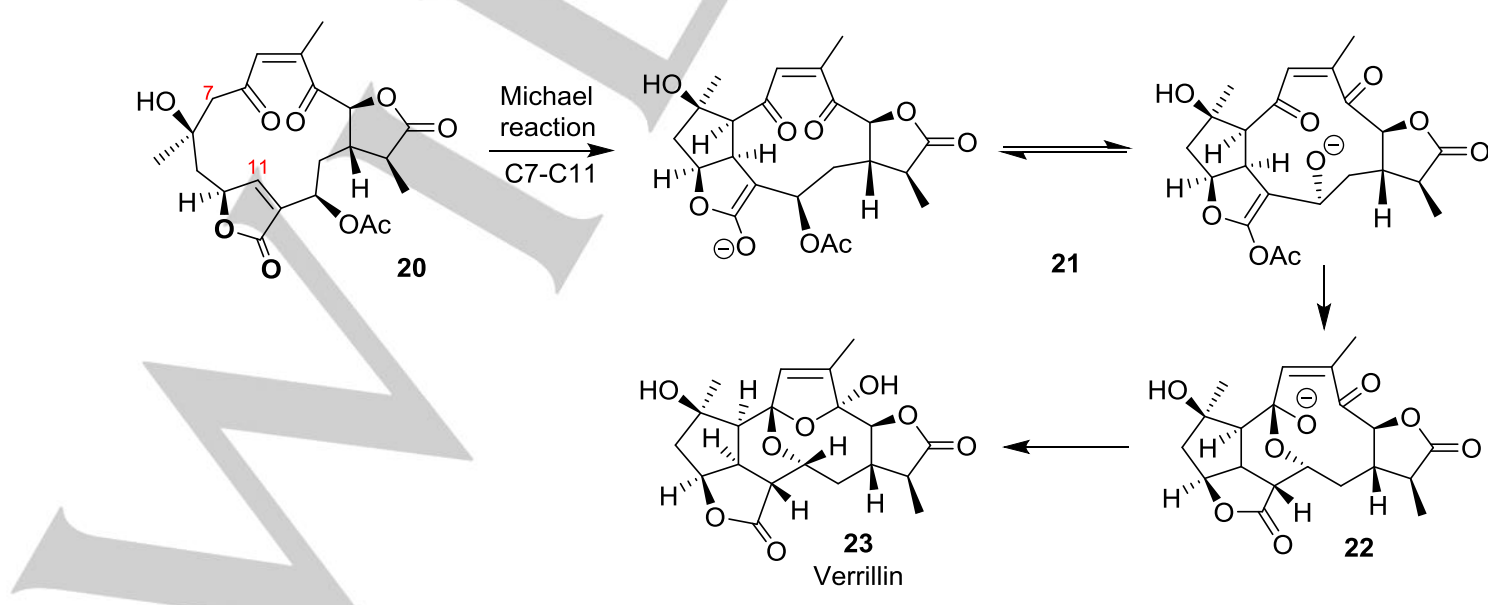




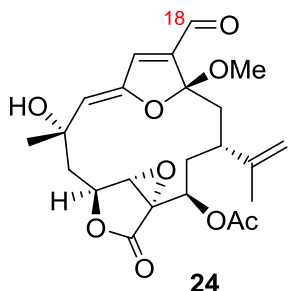

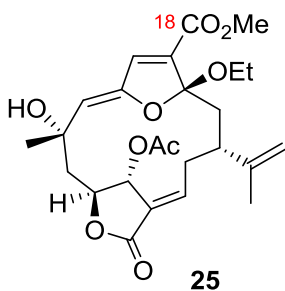

Figure 3. Some enol ether cyclic ketal metabolites isolated from corals.

Verrillin 23 co-occurs with bipinnatin $\mathrm{J}(3)$ in $P$. bipinnata ${ }^{[15]}$ and even though it has a different oxidation pattern at $\mathrm{C} 17$ and is epimeric at C8 with beilschowskysin $\mathbf{8}$, it is likely that it is also derived from an enedione intermediate similar to 17, viz 20 which, after an intramolecular Michael reaction to $\mathbf{2 1}$ followed by transacetalisation to $\mathbf{2 2}$ would cascade to verrillin (Scheme 3). Interestingly, Theodorakis et al. ${ }^{[16]}$ have recently synthesised a macrocyclic structure which accommodates the important fused cyclopentane-butyrolactone unit in $\mathbf{2 1}$ but with the enedione unit masked as a furan ring. However this biosynthesis inspired synthesis approach to verrillin has not been progressed.

Secondary metabolites which accommodate the interesting enol ether cyclic hemiketal unit in the penultimate intermediate 16 in the proposed biosynthesis of bielschowskysin 8 (see Scheme 1) have been isolated from corals, often alongside their likely C7,C8 vicinal diol and epoxide precursors. Significantly however these natural products, e.g. 24 and 25, have only been characterised with $\mathrm{CO}_{2} \mathrm{Me}$ or $\mathrm{CHO}$ groups at $\mathrm{C} 18$ (i.e. not Me as in bielschowskysin), and only as C3 methyl or ethyl ethers. In early model studies of the oxidation of vinyfurans, e.g. 26, to enol ether cyclic ketals $\mathbf{2 8}$, cf. $\mathbf{2 4}$, via the epoxide intermediate 27 it was demonstrated ${ }^{[17]}$ how crucial it was to have an ester group at C3 in the furan ring and how sensitive the enol ether cyclic ketal product $\mathbf{2 8}$ was to rearrangement/re-aromatisation

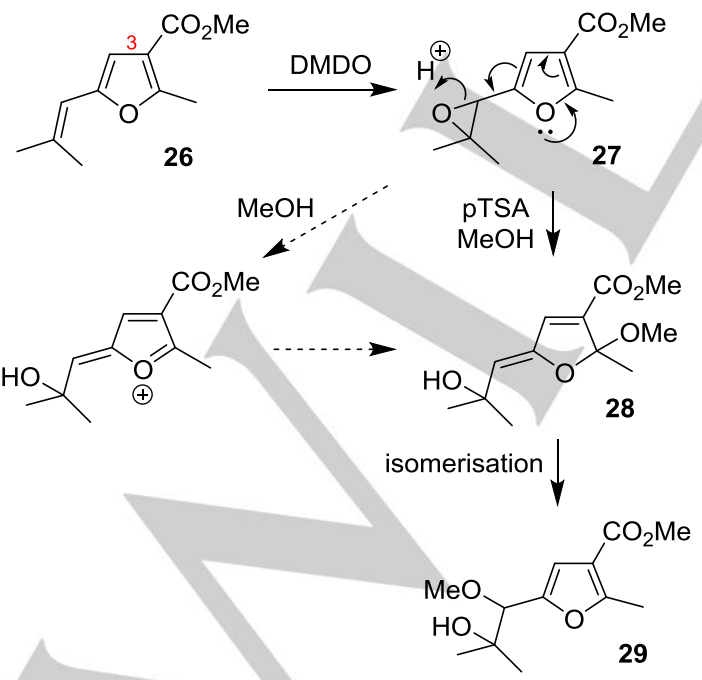

Scheme 4. A model study of the synthesis of enol ether cyclic ketals 28 from epoxy furans $\mathbf{2 7}$ in the presence of protic acids. to the more stable furan isomer, viz 29 (Scheme 4). Indeed, the enol ether cyclic ketal $\mathbf{2 8}$ was only observed and characterised by ${ }^{1} \mathrm{H}$ NMR spectroscopy before it rearranged to 29 , thus precluding any study of its [2+2] photocycloaddition chemistry. However, in parallel investigations Nicolaou et al. ${ }^{[18]}$ were able to achieve a synthesis of the macrocyclic ester-stabilised enol ether cyclic ketal compound $\mathbf{3 0}$ and then showed that it underwent facile intramolecular [2+2] photocycloaddition to produce the cyclobutane ring-fused structure $\mathbf{3 1}$ in high yield. Furthermore, some pioneering intramolecular [2+2] photochemical studies by Sullikowski et al. ${ }^{[19]}$ with substituted ylidene butenolide precursors, e.g. 32 resulted in the elaboration of several of the critical elements of the cyclobutane ring-fused system 33 in bielshowskysin. Each of the aforementioned investigations therefore combined to give some credence to the earlier biosynthetic proposal to bielschowskysin 8 involving enol ether intermediates and [2+2] photocycloaddition (see Scheme 1)
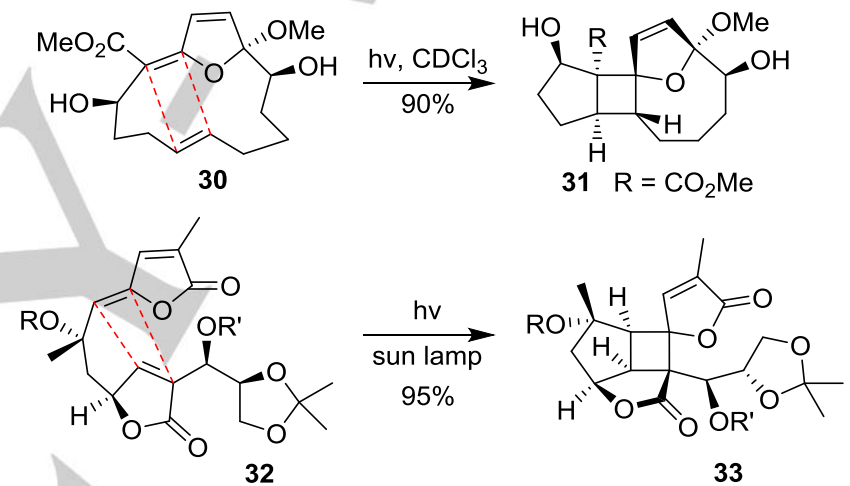

Scheme 5. Model photochemical studies towards the cyclobutane ring-fused system in bielschowskysin 8

Nevertheless, throughout the past decade synthetic chemists have concentrated their efforts towards bielschowskysin using non-biomimetic approaches, where both thermal and photochemical approaches to cyclobutane ring intermediates have all been carried out early-on in the synthetic designs. Most of these synthetic approaches to bielschowskysin, which are summarised elsewhere, ${ }^{[14]}$ were thwarted however by the difficulties in achieving satisfactory macrocyclisation procedures from substituted cylcobutane ring-containing precursors. Interestingly, in some complementary investigations of the formation of the cyclobutane ring in bielschowskysin using quantum chemical calculations Tang et al. ${ }^{[20]}$ concluded that whilst the photochemical pathway is computed to be highly efficient an alternative thermal process is also feasible in water via an unusual concerted ring-forming transition state without the intervention of an enzyme.

Moving forward from our own early model work, Roche et al. ${ }^{[21]}$ took up the challenge of establishing the earlier described biosynthetic proposal to bielschowskysin 8 by examining a synthesis of the enol ether cyclic ketal $\mathbf{3 7}$ produced from the natural product acerosolide $\mathbf{5}$ and then studying its 

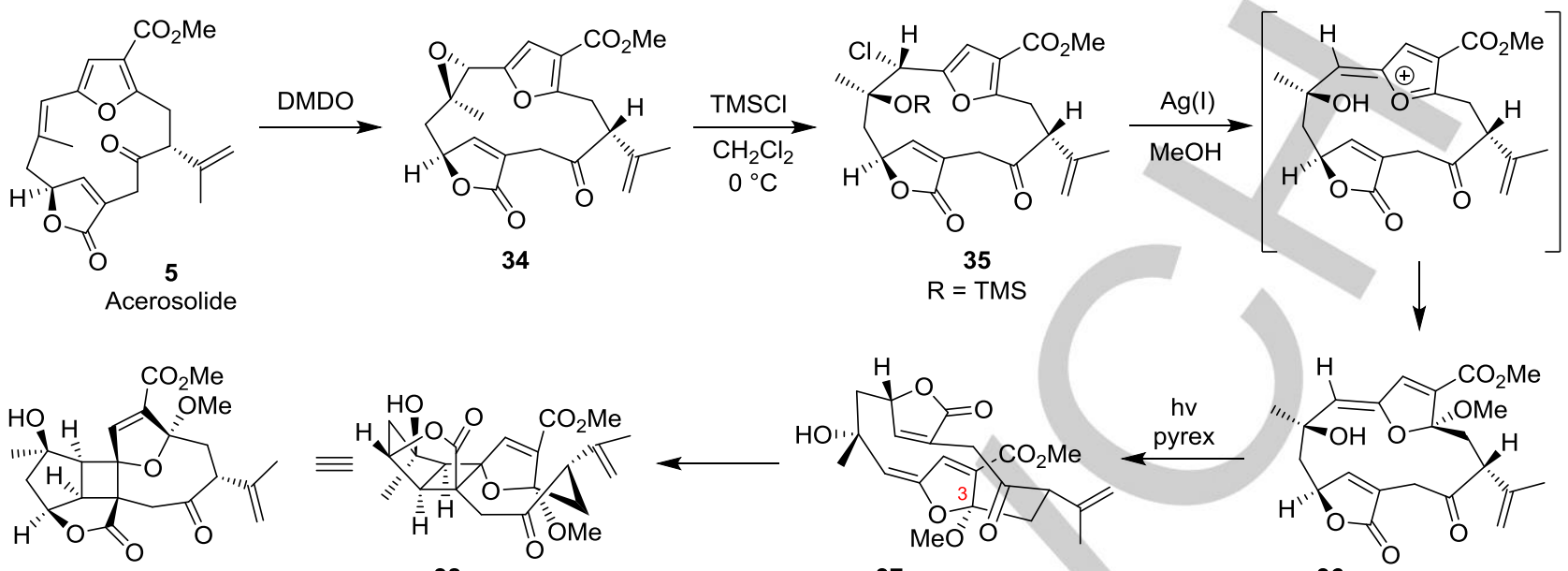

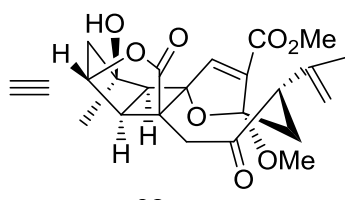

38

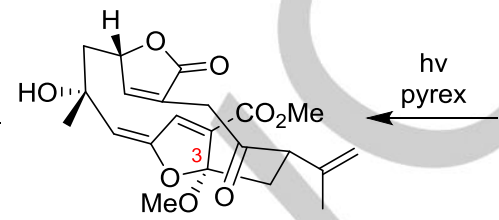

37

E-isomer

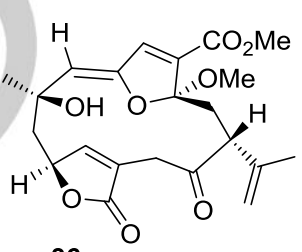

36

Z-isome

Scheme 6. Synthesis of the macrocyclic Z-enol ether cyclic ketal $\mathbf{3 6}$ from acerosolide $\mathbf{5}$ followed by its isomerisation to $\mathbf{3 7}$ and intramolecular photochemical [2+2] photocycloaddition to the bielschowskysin analogue $\mathbf{3 8}$.

intramolecular [2+2] photocycloaddition to the cyclobutane ring in the analogue $\mathbf{3 8}$ of bielschowskysin. Key to their success was the conversion of the epoxide $\mathbf{3 4}$ derived from acerosolide into the labile chlorohydrin 35 following treatment with $\mathrm{TMSCl}$ (Scheme 6). Halogen abstraction from the chlorohydrin 35 mediated by a $\mathrm{Ag}(\mathrm{I})$ salt, next led to the furanoxonium ion intermediate shown, which was intercepted under kinetic control at $-78{ }^{\circ} \mathrm{C}$ by methanol to give the $Z$ - enol ether cyclic ketal 36 (46\% from the epoxide 34 ). The $Z$-enol ether cyclic ketal 36 was found to be stable for three days in the dark, but when a solution in chloroform was irradiated at $254 \mathrm{~nm}$ through Pyrex it was isomerised to the $E$-isomer $\mathbf{3 7}$ which underwent intramolecular [2+2] cycloaddition leading to the polycycle 38 containing the bielschowsksin ring system. None of the synthetic steps to 38 were trivial, and the stereochemistry at the ketal C3 centre in $\mathbf{3 7}$ was shown to be critical to realise the final intramolecular [2+2] photochemical cycloaddition. Unlike bipinnatin $J(3)$, the proposed biosynthetic precursor to bielschowsksin, acerosolide 5 has a $\mathrm{CO}_{2} \mathrm{Me}$ rather than a Me group at $\mathrm{C} 4$, together with an $E$-rather than $Z$ - double bond at $\mathrm{C} 7, \mathrm{C} 8$ and an additional carbonyl group at C14. Nevertheless, the study by Roche et al. ${ }^{[21]}$ provides a significant blueprint for the biosynthesis of bielschowsksin 8 via an intramolecular [2+2] cycloaddition from an enol ether cyclic ketal intermediate as key step. We await further developments of this exciting biomimetic synthetic approach to bielschowskysin 8 .

\section{Plumarellide and rameswaralide}

Plumarellide 9 was isolated from the gorgonian coral Plumarella $\mathrm{sp}$ which inhabits the Kuri Island region of the Pacific ocean, in $2002 .{ }^{[8]}$ It is characterised by having a central cyclohexene ring which is conjoint with a substituted cyclopentane and an oxy- bridged cycloheptene. The metabolite is structurally related to the earlier isolated mandapamates $39^{[22]}$ and $40^{[23]}$ found in soft corals of the genus Sinularia which are epimeric at $\mathrm{C} 8$ and have a $\mathrm{CO}_{2} \mathrm{Me}$ group instead of a $\mathrm{CH}_{2} \mathrm{OH}$ at $\mathrm{C} 18$ in their structures (Fig 4). Rameswaralide 10, by contrast, has a central cycloheptene ring flanked by substituted cyclopentane and cyclohexene rings. $^{[9]}$ It co-occurs with the mandapamates 39 and $\mathbf{4 0}$ in Sinularia dissecta, and it was earlier suggested that rameswaralide could be derived via ring expansion of the 6 membered ring in a mandapamate via a novel vinylogous a-ketol rearrangement. ${ }^{[1]}$ The four metabolites $9,10,39$ and 40, show subtle variations in the orientations of the $\mathrm{C} 7$ and $\mathrm{C} 8$ centres in their structures. Thus, whereas plumarellide 9 has an $\alpha$ orientated $\mathrm{OH}$ group at $\mathrm{C} 8$, the same $\mathrm{OH}$ group in the

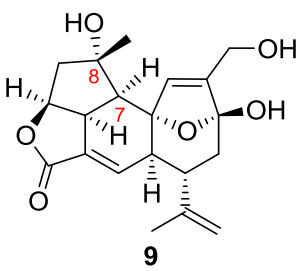

Plumarellide

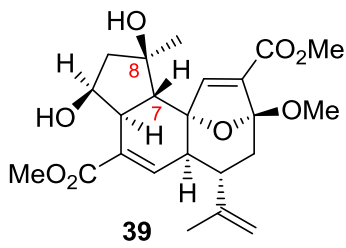

Mandapamate<smiles>C=C(C)[C@H]1CC(O)=C(C(C)=O)[C@@H]2C(=O)C3[C@H](O)C[C@H]4OC(=O)C(=C[C@@H]32)[C@@H]14</smiles>

Rameswaralide

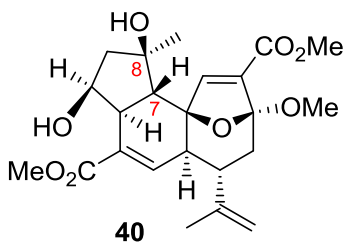

Isomandapamate
Figure 4. Natural products plumarellide 9, rameswaralide $\mathbf{1 0}$ and the mandapamates $\mathbf{3 9}$ and $\mathbf{4 0}$ 
metabolites 10,39 , and 40 is orientated $\beta$ instead. Also, whereas the bridged $\mathrm{C} 7-\mathrm{H}$ centre in $\mathbf{9}$ and $\mathbf{1 0}$ is $\alpha$-orientated, the same centre in the mandapamates $\mathbf{3 9}$ and $\mathbf{4 0}$ has the alternative $\beta-\mathrm{H}$ stereochemistry. These differences clearly have significanceregarding the biosynthetic origins of the four metabolites.

It has been proposed that the fused ring systems in plumarellide $\mathbf{9}$ and rameswaralide $\mathbf{1 0}$ have their origins in FBC precursors, viz 41 and 45 which have unsaturation at C13-C14, by way of conversion into enol ether cyclic hemiketal intermediates, e.g 42 (cf bielshowsksin biosynthesis earlier) and/or enedone intermediates, viz 46 , followed by transannular $(4+2)$ and $(4+3)$ type cycloaddition reactions respectively, viz $\mathbf{4 2} \rightarrow$ plumarellide $\mathbf{9}$, and 48 $\rightarrow$ rameswaralide 10 (Schemes 7 and 8). ${ }^{[1,2]}$ Alternative two-step ionic pathways to the same metabolites have also been proposed involving 5-exo trig cyclisation between $\mathrm{C} 7$ and $\mathrm{C} 11$ in the enedione tautomers $\mathbf{4 3}$ (for plumarellide) and 47 (for rameswaralide) (Schemes 7 and 8 ) followed by intramolecular vinylogous aldol reactions from the enolate intermediates $\mathbf{4 4}$ and 48 respectively. ${ }^{[1]}$ The cyclohexene rings in the mandapamates 39 and $\mathbf{4 0}$ are thought to arise by way of a similar intramolecular $(4+2)$ processes to that shown for plumarellide.

More recently, in 2016, Chitturi et al. ${ }^{[2]}$ described the "pambanolide" structures 50 and 51 which were isolated alongside rameswaralide $\mathbf{1 0}$ and mandapamate $\mathbf{3 9}$ from the soft coral Sinularia inelegans. The authors suggested that pambanolide $A(\mathbf{5 0})$ could be derived from mandapamate 39 in vivo following a reverse vinylogous aldol reaction leading to the same intermediate 49 proposed in Scheme 8 to ramesawaralide 10. It seems more likely however that the natural product is

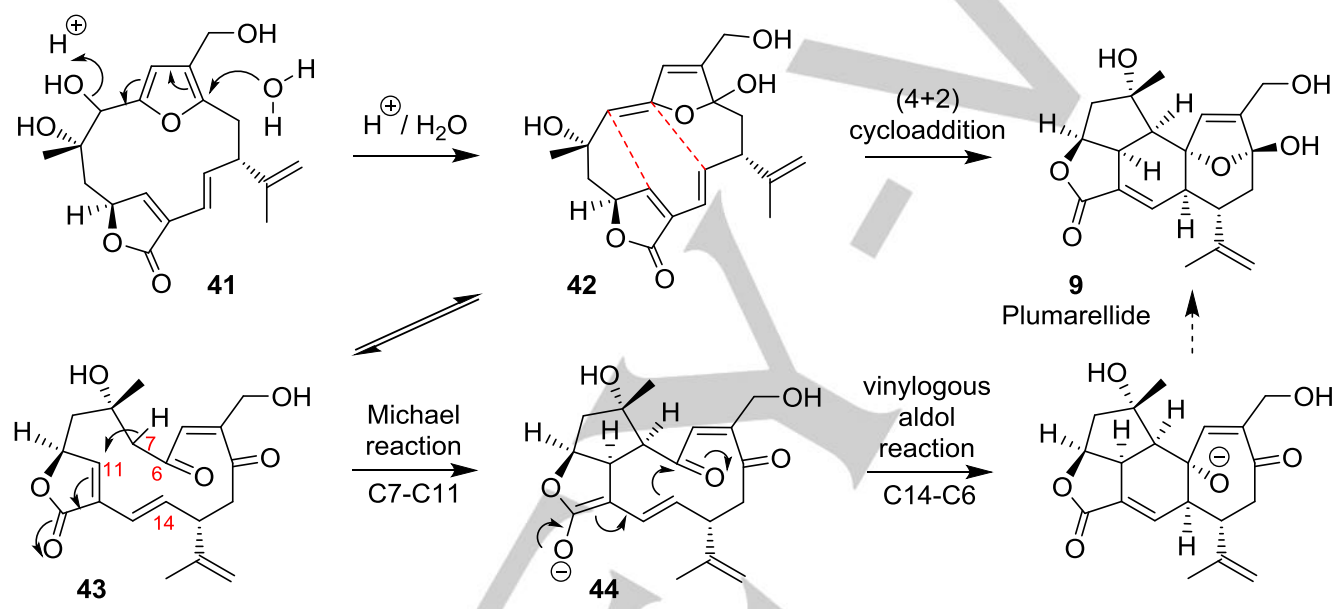

Scheme 7. Proposals for the biosynthesis of plumarellide $\mathbf{9}$ from the macrocyclic enol ether cyclic hemiketal $\mathbf{4 2}$ via intramolecular (4+2) type cycloaddition or via a two-step ionic process from the enedione $\mathbf{4 3}$, viz. $\mathbf{4 3 \rightarrow 4 4 \rightarrow 9}$

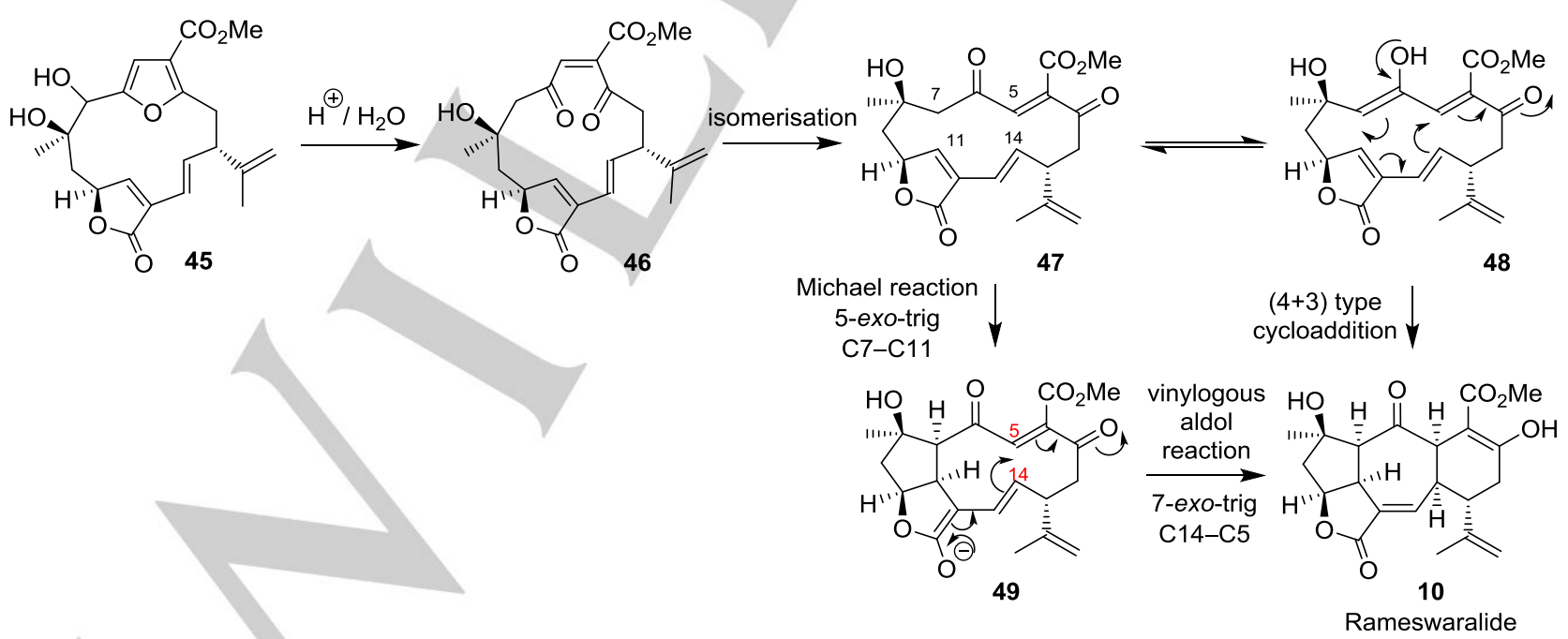

Scheme 8. Proposals for the biosynthesis of rameswaralide $\mathbf{1 0}$ from the macrocyclic polyene dione $\mathbf{4 7}$ via intramolecular (4+3) type cycloaddition or via a two step ionic process, viz. $\mathbf{4 7} \rightarrow \mathbf{4 9} \rightarrow \mathbf{1 0}$. 


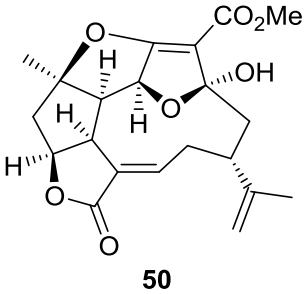

Pambanolide A

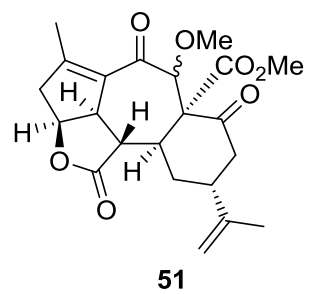

Pambanolide B
Figure 5. Structures of the pambanolides $\mathbf{5 1}$ and $\mathbf{5 2}$ from Sinularia inelegans.

derived from the FBC $\mathbf{4 5}$ via $\mathbf{4 9}$ followed by isomerisation to the $E$ - enedione $\mathbf{5 2}$ and cyclisation to the bridged ether intermediate 54 (Scheme 9). Cyclic hemiketal formation from $\mathbf{5 4}$ would next lead to $\mathbf{5 3}$, the positional isomer of pambanolide $A(\mathbf{5 0})$. The intermediate $\mathbf{5 2}$ is also the likely precursor to pambanolide $B$ (51) in S. elegans by addition of methanol at C5 triggering a Michael cyclisation across C4 and C13 leading to 55 which then eliminates water producing $\mathbf{5 1}$ (Scheme 10). Up until very recently a perplexing issue with the aforementioned biosynthetic proposals for plumarellide, rameswaralide and the mandapamates was that no FBC epoxide or similar precursor having 1,3-diene functionality between $\mathrm{C} 11$ and $\mathrm{C} 14$ had been described in corals. This changed in 2018 when Baker et al. ${ }^{[25]}$ uncovered the keikipukalides 56 and 57 , displaying such unsaturation, in the deep sea octocoral Plumarella delicatissima collected from the "Plateau of Fascination" north east of Stanley, Falkland Islands in the Southern Ocean. The characterisation of the keikipukalides has given some credence to, and confidence in the broad proposals put forward in Schemes 7 and 8 to plumarellide $\mathbf{9}$ and rameswaralide $\mathbf{1 0}$.

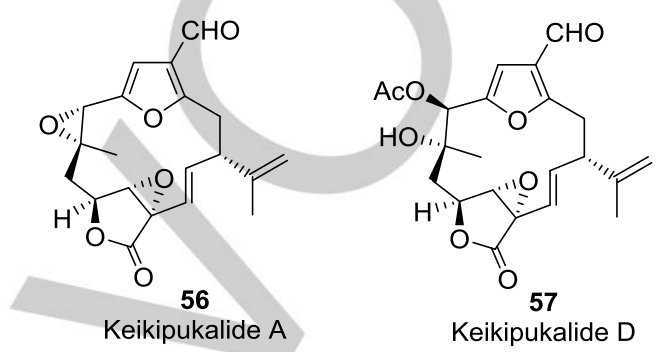

Figure 6. Structures of the keikipukalides 56 and 57 found in Plumarella delicatissima.

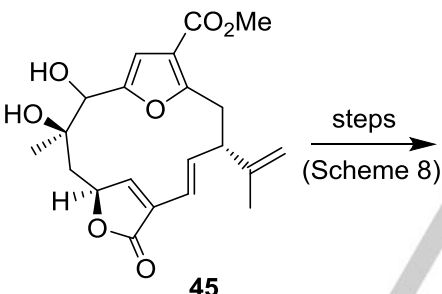

45

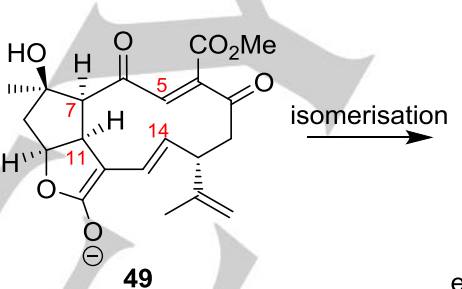

49

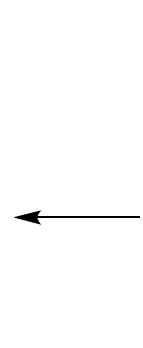

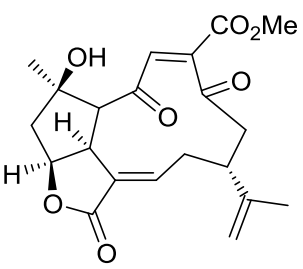

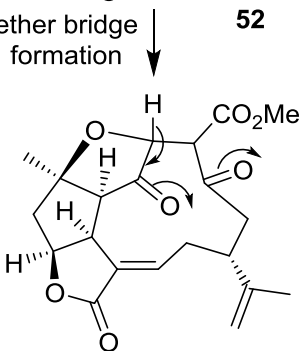

54

Pambanolide A

Scheme 9. Proposal for the biosynthesis of pambanolide A (50) from the FBC $\mathbf{4 5}$ via the enedione $\mathbf{5 2}$ and the cyclic ether $\mathbf{5 4}$ intermediates.

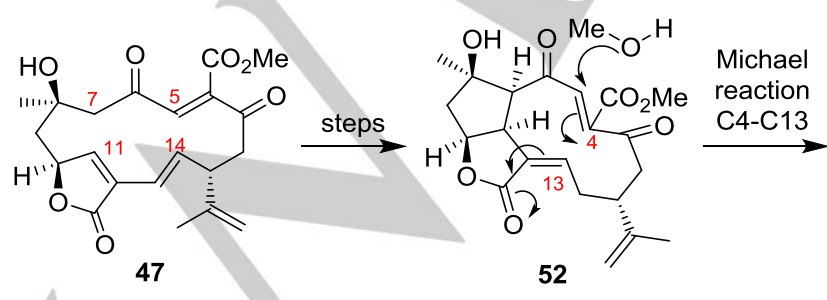

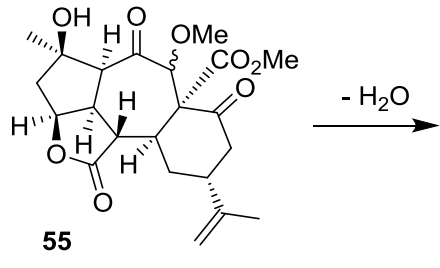

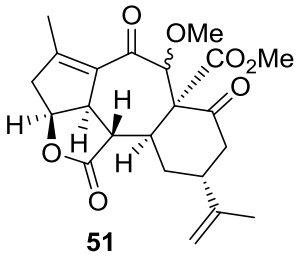

Pambanolide B

Scheme 10. Proposal for the biosynthesis of pambanolide B (51) from the enedione $\mathbf{4 7}$ via the tricylic intermediate $\mathbf{5 5}$ implicating concerted methanol addition and an intramolecular Michael reaction. 


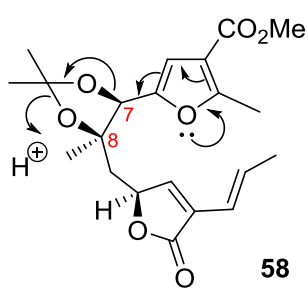<smiles>CC(C)(C)OC(=O)OC(C)(C)C</smiles>

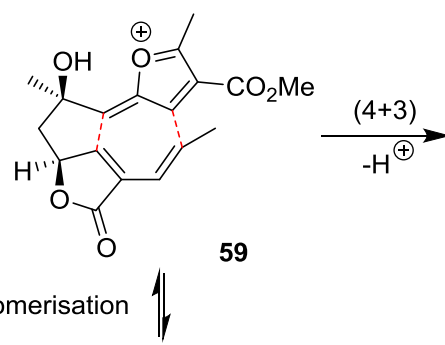

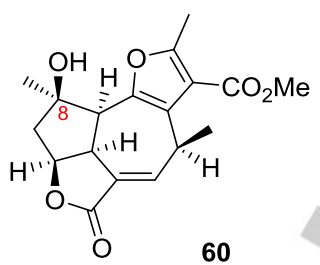

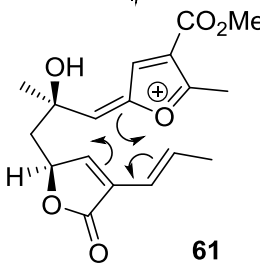

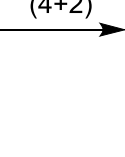

\section{$\mathrm{H}$,}

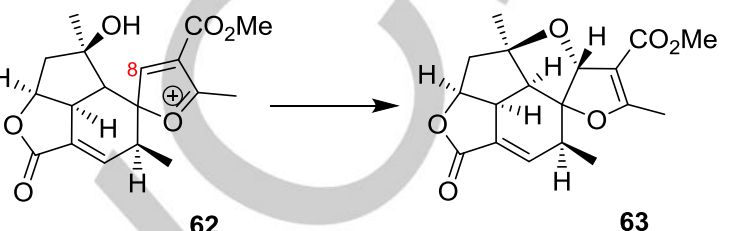

Scheme 11. Formation of the rameswaralide and plumarellide ring systems 60 and 63 respectively from acid treatment of the model furanobutenolide 58 implicating (4+3) and (4+2) type cycloadditions.

In pursuit of a unified biosynthetic approach to plumarellide and rameswaralide based on the proposals in Schemes 7 and 8, Pattenden and his group ${ }^{[26-28,30-31]}$ made an extensive study of the acid-catalysed transformations of the model $\mathrm{C} 8 \mathrm{OH}$ diastereoisomeric furanobutenolide acetonide isomers 58 and 64. When the $\beta$-orientated $\mathrm{C} 8 \mathrm{OH}$ diastereoisomer 58 was treated with TFA in DCM it was hydrolysed to the $E$-and $Z$ isomeric furanoxonium ion intermediates 59 and 61 which then underwent separate transannular cyclisations producing a 7:5 mixture of the polycycles 60 and 63 containing the rameswaralide and plumarellide ring systems respectively in a combined $60 \%$ yield (Scheme 11). Perhaps not too surprisingly, treatment of the diastereoisomer $\mathbf{6 4}$ of $\mathbf{5 8}$, having the alternative a-orientated $\mathrm{OH}$ at $\mathrm{C} 8$, under similar conditions gave only the $\mathrm{C} 8$ epimer 65 of the rameswaralide ring system 60 (with no plumarellide ring- containing by-product) (Scheme 12). It was proposed that formation of the diastereoisomeric rameswaralide ring-containing compounds 60 and 65 resulted from unprecedented $(4+3)$ type cycloaddition reactions from furanoxonium ion intermediates, viz 59. At the time these studies were carried out they were the first demonstrations of

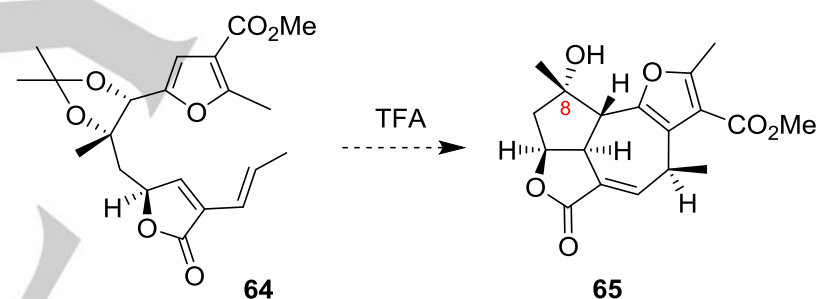

Scheme 12. Formation of the rameswaralide ring system 65 from acid treatment of the model furanobutenolide 64

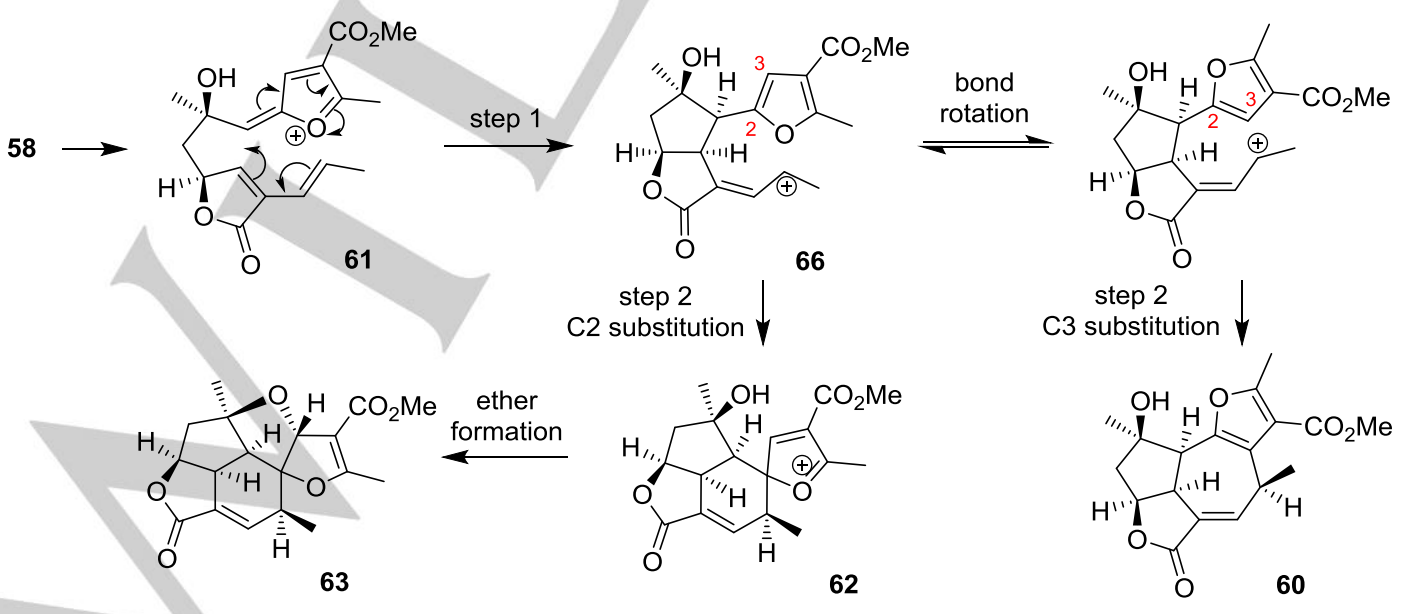

Scheme 13. Two-step carbonium ion cyclisation pathways for the conversion of the furanobutenolide $\mathbf{5 8}$ into the rameswaralide and plumarellide ring systems $\mathbf{6 0}$ and 63 respectively. 

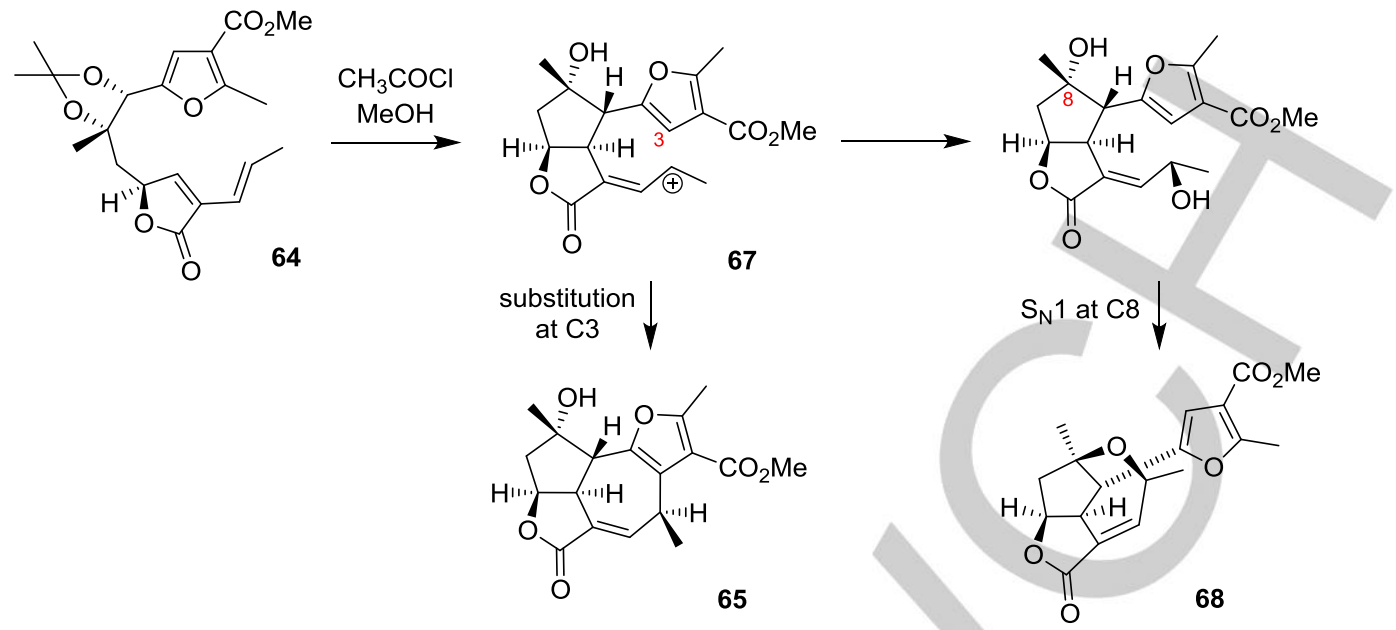

Scheme 14. Rationale for the formation of the 7-ring cyclic ether by-product 68 following treatment of the acetonide 64 with $\mathrm{HCl}$.

furanoxonium ions taking part in $(4+3)$ cycloadditions. Later, Johan Winne et al. and other researchers greatly expanded the scope of this novel $(4+3)$ cycloaddition process to the synthesis of different, related ring systems. ${ }^{[29,30]}$

The isolation of the cyclohexene plumarellide ring-containing compound 63, (albeit "strapped" via an ether bridge), from acid treatment of the model furanobutenolide acetonide $\mathbf{5 8}$ gave some support to the $(4+2)$ cycloaddition biosynthetic rational towards plumarellide put forward in Scheme 7. However it was also suggested that the conversion $\mathbf{5 8} \rightarrow \mathbf{6 3}$ might proceed by an alternative stepwise carbocation ion cyclisation sequence, i.e. $61 \rightarrow 66$ followed by $66 \rightarrow 62$ as shown in Scheme $13 .{ }^{[28]}$ Likewise, the conversion of $\mathbf{5 8}$ into the rameswaralide ring-containing system $\mathbf{6 0}$ could also be represented as a two-step carbonium ion cyclisation sequence, i.e. $\mathbf{6 1} \rightarrow \mathbf{6 6}$ followed by $\mathbf{6 6} \rightarrow \mathbf{6 0}$, rather than a $(4+3)$ type cyclisation (Scheme 13). In an experiment, which gave some credence to this proposal, the diastereoisomeric acetonide $\mathbf{6 4}$ was treated with acetyl chloride- methanol (to generate $\mathrm{HCl}$ ) in DCM and the presumed firstformed allylic carbocation intermediate 67 [cf. 66 Scheme 13] was intercepted leading to the interesting 7 -ring ether 68 in $20 \%$ yield (Scheme 14) in addition to the expected rameswaralide ring-containing polycycle 65 . $^{[27,31]}$

In complementary DFT calculation studies, ${ }^{[32]}$ aimed at probing $(4+2)$ and $(4+3)$ intramolecular cycloadditions involving furanoxonium ion intermediates, the suggested alternative stepwise carbonium ion cyclisation reactions shown above, to plumarellide and to rameswaralide models 63 and 60 (Scheme 13), were shown to be highly probable, and indeed favour the products formed. DFT calculations were also made to compare the stepwise cyclisation pathway with the [4+2] cyclisation of the furanoxonium ion intermediate $\mathbf{7 0}$ produced from the macrocyclic FBC 69 leading to the tetracyclic ring system $\mathbf{7 5}$ present in plumarellide 9 (Scheme 15). In addition, since the cyclisation $\mathbf{7 0} \rightarrow \mathbf{7 5}$ could also take place by stepwise acidcatalysed cyclisation from the enedione tautomer 73 of 71 via

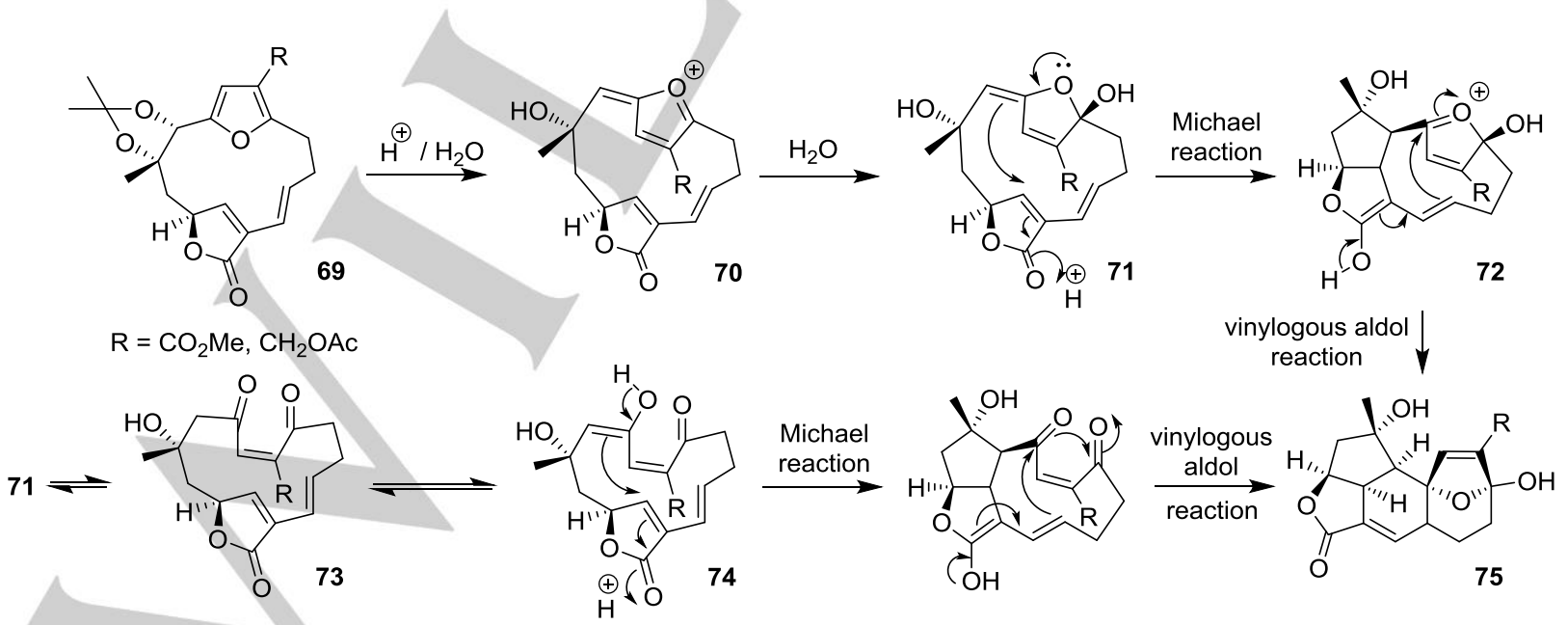



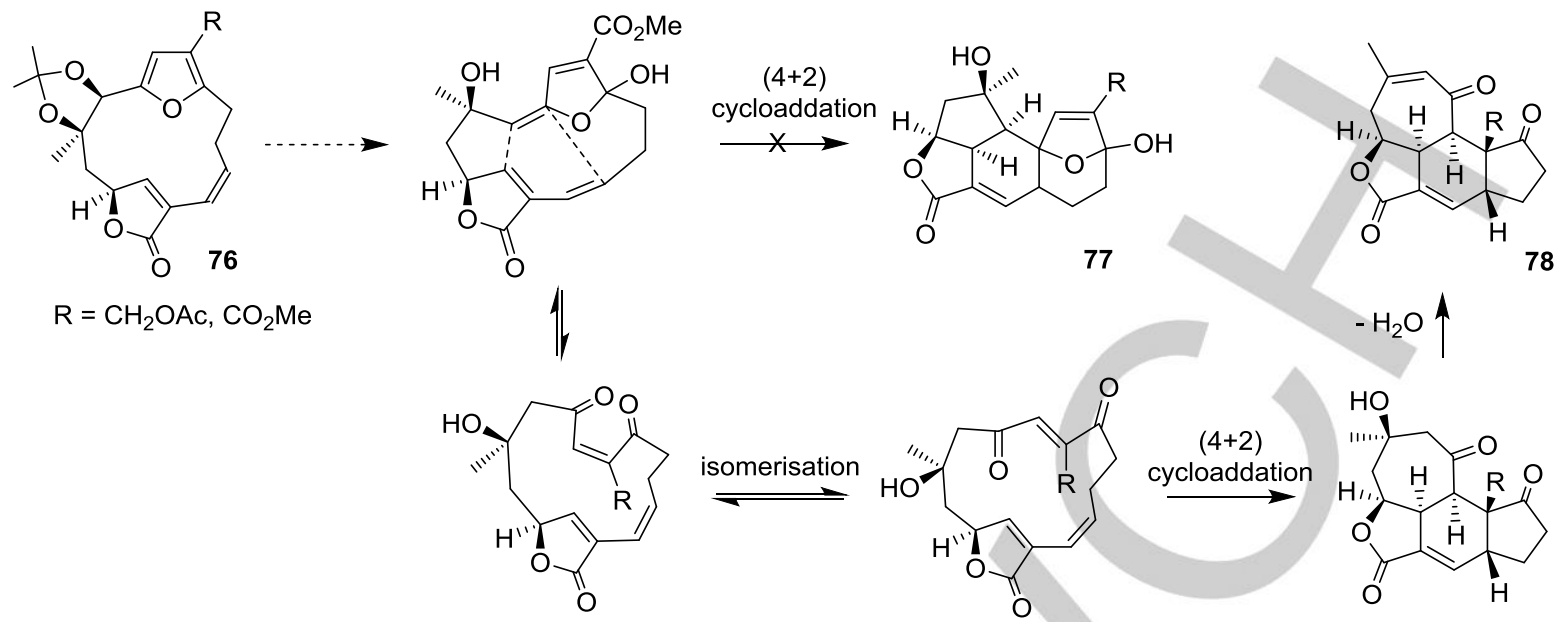

Scheme 16. Formation of the polycyclic structure 78 in preference to the plumarellide ring system 77 following acid-catalysed rearrangement of the $\mathrm{C} 13$, C14 unsaturated furanobutenolide-based acetonide 76 .

the enol 74, these alternative cyclisation possibilities were also investigated. Overall, the DFT calculation studies predicted that the acid-catalysed stepwise carbonium ion pathway $\mathbf{7 1 \rightarrow 7 2 \rightarrow 7 5}$ was more favourable than the thermal [4+2] cycloaddition. Furthermore, the enedione $\mathbf{7 3}$ was found to be relatively more stable than its cyclic hemiacetal tautomer $\mathbf{7 1}$ which is relatively more stable than its enol tautomer $\mathbf{7 4}$.

In other studies which were designed to achieve a biomimetictype synthesis of the plumarellide ring system 77 , the Z-olefin isomer of the macrocycle 76 corresponding to 69 was synthesised and then treated with TFA. ${ }^{[33]}$ Disappointingly, this experiment gave none of the plumarellide structure 77 and instead produced the interesting polycyclic structure 78 in $82 \%$ yield. The structure 78 is thought to arise from 76 by hydrolysis leading to an enol ether cyclic hemiketal intermediate followed by an intramolecular $(4+2)$ cycloaddition process from its enedione tautomer, as shown in Scheme $16 .^{[33]}$

The aforementioned studies, inspired by biosynthesis speculation, clearly provided a wealth of new synthesis and mechanistic knowledge on the complex pathways to the ring systems in plumarellide and rameswaralide implicating furanoxonium ions and their derivatives as central intermediates. Nevertheless, the studies are incomplete and further work by those who are excited by the challenge of designing and then evaluating biomimetic synthesis must come forward.

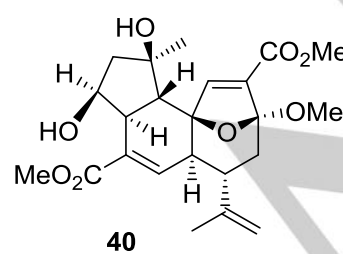

Isomandapamate

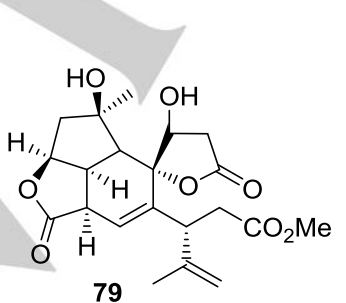

Havellockate
Figure 7. Proposed origin of havellockate $\mathbf{7 9}$ form isomandapamate $\mathbf{4 0}$
Over the past two decades a number of complementary in vitro approaches have been made towards the synthesis of the ring systems in the natural products $9,10,39$ and 40 , and also to the related seco-cembranoid structure havellockate $79,{ }^{[34]}$ which is likely to be derived from isomandapamate in Sinularia granosa. (Figure 7) Interested readers should study a recent comprehensive review of these synthetic approaches. ${ }^{[14]}$

\section{Intricarene}

Intricarene 11 was isolated alongside bielschowskysin 8 from the coral Pseudopterogorgia kallos by Rodrigues et al. in 2005. ${ }^{[10]}$ An hypothesis for its biosynthesis from bipinnatin $\mathrm{J}(3)$ was put forward simultaneously by Trauner $^{[35]}$ and by Pattenden ${ }^{[36]}$ which envisaged oxidative cleavage of the furan ring in bipinnatin $\mathbf{J}$ leading to the enedione $\mathbf{8 0}$ which should exist as the hydroxypyranone tautomer 81a. Elimination of water from 81a would next lead to the oxidopyrylium intermediate $\mathbf{8 2}$ which would be expected to undergo intramolecular dipolar [5+2] cycloaddition leading to intricarene 11 (Scheme 17). This proposed biosynthetic pathway was mimicked in vitro by the groups of both Trauner and Pattenden in almost identical fashion, and published in 2006. ${ }^{[35,36]}$ Both research groups developed asymmetric syntheses of bipinnatin $\mathrm{J}(3)$ which they next oxidised using different oxidants to the hydroxyl pyranone 81a, characterised as its acetate $\mathbf{8 1 b}$. Exposure of the acetate 81b to DBU in refluxing acetonitrile (Pattenden) or to TMP in DMSO at $150{ }^{\circ} \mathrm{C}$ (Trauner) then produced intricarene in $10-26 \%$ yield. Tantillo et al. ${ }^{[37]}$ later provided computational evidence that the key cycloaddition step $\mathbf{8 2} \rightarrow \mathbf{1 1}$ was feasible thermally with an activation barrier of approx. $20 \mathrm{kcal} / \mathrm{mol}^{-1}$. On this basis therefore the aforementioned in vitro syntheses of intricarene, in hot refluxing solvents, could hardly be presented as "biomimetic". 
Later, in 2014 Trauner and colleagues, ${ }^{[38]}$ made some interesting studies of the photochemistry of the enedione 84 which they produced from oxidation of the O-methyl ether 83 of bipinnatin $\mathrm{J}$ (3). Irradiation of a solution of $\mathbf{8 4}$ in 1:1 $\mathrm{H}_{2} \mathrm{O}$-deuterochloroform using ultraviolet light from a common reptile lamp remarkably led to intricarene 11 (25\%) alongside the hydroxyl cyclopentenone 89. Quantum chemical calculations provided an insight into the likely photochemical pathway from bipinnatin $\mathrm{J}$ methyl ether to intricarene and implicated the biradical intermediates 85, 86 and 87 (Scheme 18). Furthermore, they showed that the formation of the diradical pyrone intermediate $\mathbf{8 6}$ and the cycloaddition occur via a triplet state implicating a stepwise 1,3-dipolar cycloaddition. The formation of the by-product $\mathbf{8 9}$ is thought to arise from $\mathbf{8 6}$ through a radical recombination to the epoxycyclopentenone $\mathbf{8 8}$ which, by vinylogous nucleophilic addition of water leads to $\mathbf{8 9}$ These interesting photochemical studies raised the question whether or not intricarene $\mathbf{1 1}$ is an actual natural product biosynthesised in the coral or whether it might be an artefact formed during the isolation procedure. To add to the debate, the O-methyl ether 83 of bipinnatin $\mathrm{J}$ has been isolated from $P$. bipinnatis and is the only $\mathrm{C} 2$ methyl ether of a furanocembranoid to be described. Perhaps this methyl ether is also an artefact, produced by methanolysis of bipinnatin $\mathrm{J}$ during isolation?

The total synthesis of intricarene described independently by Trauner and by Pattenden in 2006 was the first demonstration of the scope for biomimetic synthesis amongst the complex polycyclic cembranoids represented by structures $\mathbf{8 - 1 4}$, and has remained a significant milestone in this area.

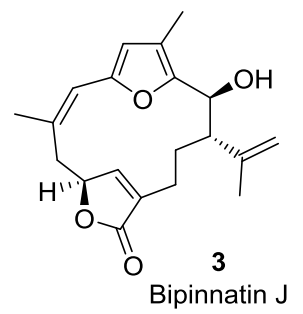

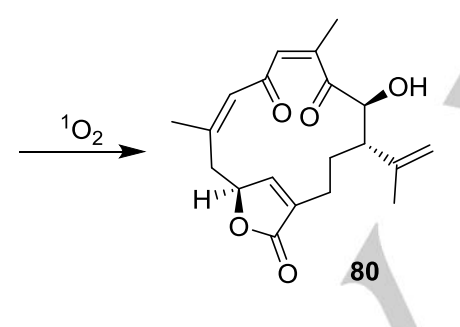

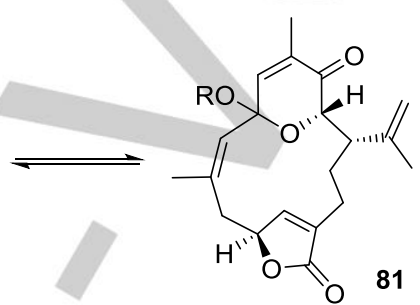

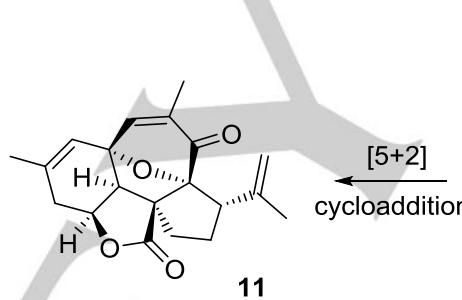

Intricarene

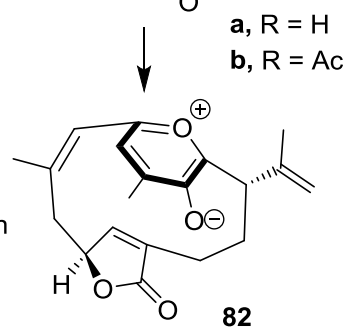

Scheme 17. Proposal for the biosynthesis of intricarene 11 involving an intramolecular dipolar [5+2] cycloaddition from the oxidopyrylium intermediate $\mathbf{8 2}$

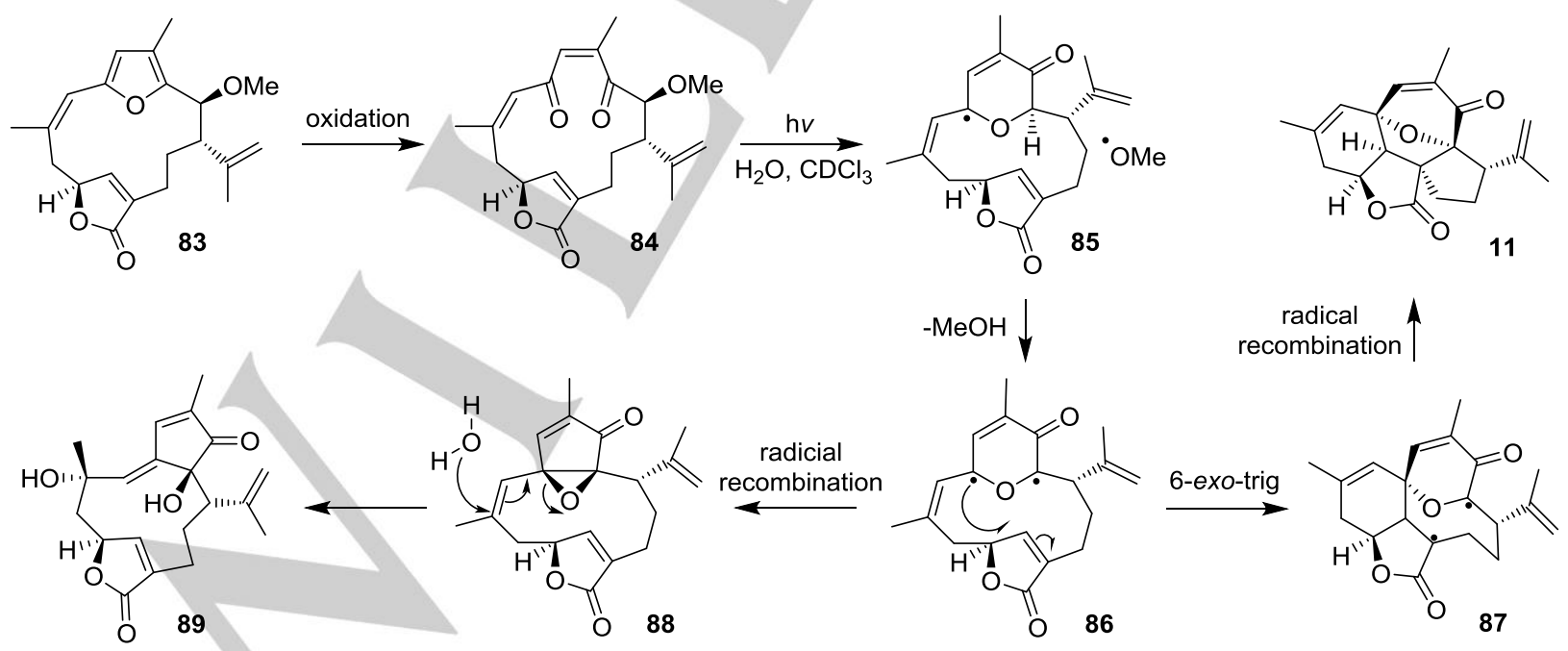

Scheme 18. A photochemical pathway to intricarene $\mathbf{1 1}$ from the methyl ether $\mathbf{8 3}$ of bipinnatin $\mathrm{J}$ implicating the biradical intermediates $\mathbf{8 5 - 8 7}$ 


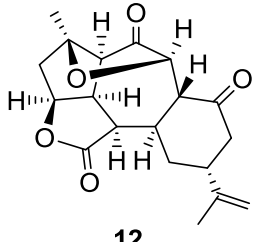

12

Ineleganolide

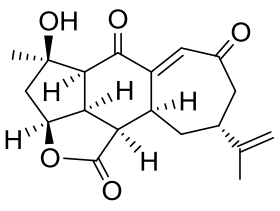

88

Scabrolide B

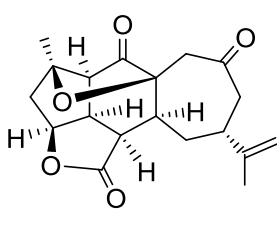

13

Sinulochmodin C

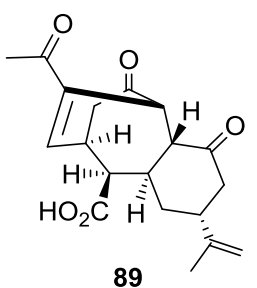

Kavaranolide<smiles>C=C(C)[C@@H]1CC(=O)C[C@@H](O)[C@]2(O)C1C=C1C(=O)O[C@H]3C[C@](C)(O)CC132</smiles>

14

Dissectolide A

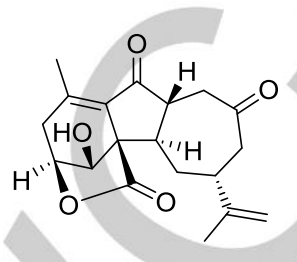

90

Sinulanocembranolide A

Figure 8. Some polycyclic C19 norcembranoids from soft corals of the genus Sinularia

\section{Ineleganolide and related norcembranoids}

Polycyclic $\mathrm{C}_{19}$-norcembranoids, represented by ineleganolide 12, ${ }^{[11]}$ sinulochmodin C (13),${ }^{[12]}$ dissectolide A (14),${ }^{[13]}$ scabrolide $\mathrm{B}(\mathbf{8 8}),{ }^{[39]}$ kavaranolide $\mathbf{8 9}^{[40]}$ and sinulanocembranolide $\mathrm{A}$ $(90),{ }^{[41]}$ are found exclusively in soft corals of the genus Sinularia. Dissectolide A (14) and the antileukemic ineleganolide 12 were amongst the first members of this family to be characterised, during the 1990s, and in the early 2000s sinulochmodin C (13) and scabrolide $B(\mathbf{8 8})$ were isolated from S.lochmodes and $S$. scabria respectively. Kavaranolide 89 and sinulanocembranolide A (90) are examples of more recently isolated norcembranoids from corals.
The polycycles in Figure 8 show several structural features in common with the $\mathrm{C}_{20}$-polycyclic cembranoids discussed earlier, and they are often found in corals alongside C19 macrocyclic $3(2 \mathrm{H})$-furanone-based norcembranoids, particularly 5episinuleptolide 95, which lack a carbon constituent, (i.e. C18) at $\mathrm{C} 4$ in their structures. The $3(2 \mathrm{H})$-furanone ring in 5episinuleptolide, and other norcembranoids, has its origins in oxidative cleavage of the furan ring in an FBC precursor, e.g. 91 followed by hydration and oxy-Michael cyclisation which first lead to 92 (Scheme 19). ${ }^{[6]}$ The C18 group R in 92 is then lost, probably following decarboxylation from the $\beta$-keto acid intermediate 93 leading to 94 . Further oxidation and rearrangement involving $\mathrm{C} 11-\mathrm{C} 13$ in 94 would then produce 5episinuleptolide $95 .^{42}$ It is no coincidence that all FBCs that

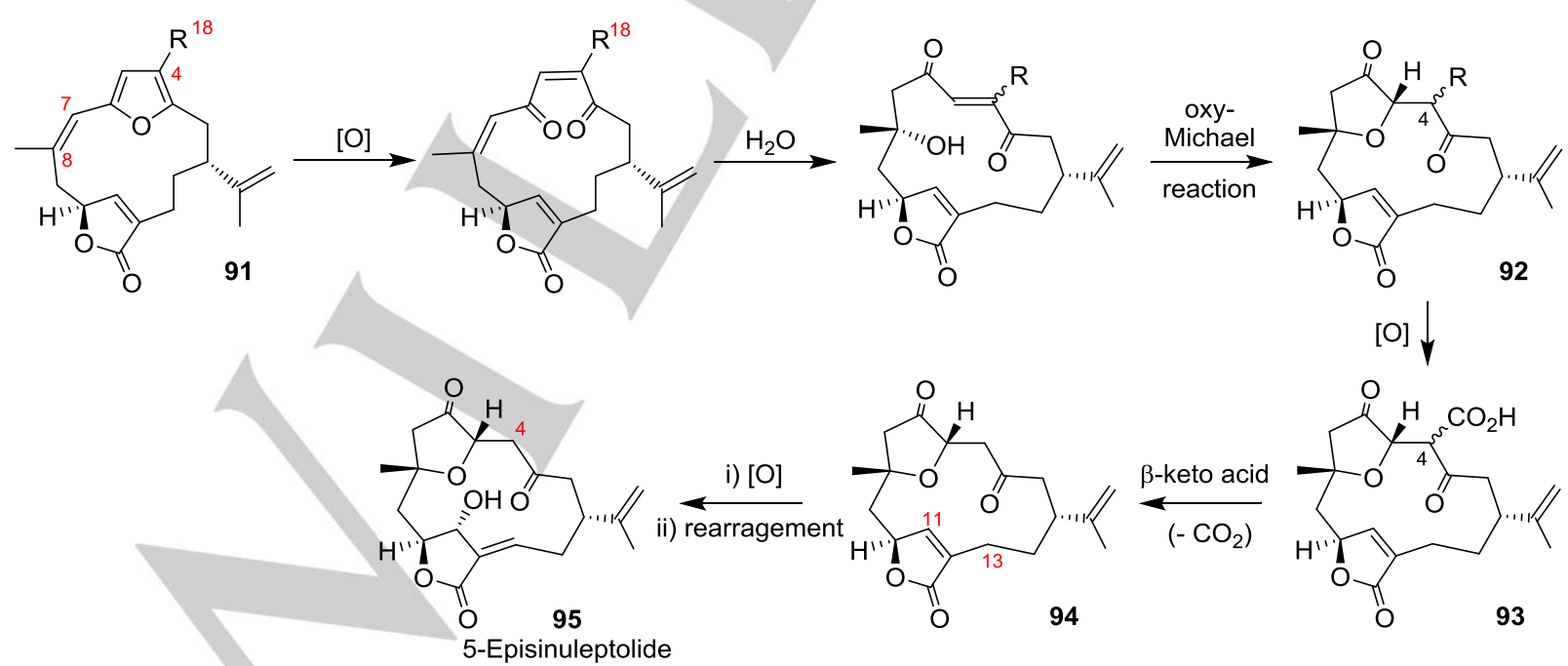

Scheme 19. Proposal for the origin of the norditerpene 5-episinuleptolide 95 from the FBC 91 involving loss of the C18 substituent in the furanone intermediate 93. 
<smiles>[R]C1=C2C=C(C)CC(=C1)C=C(C[C@@H](CCC1=CCOC1=O)C(=C)C)O2</smiles>

i) $\mathrm{OsO}_{4}$, $\underset{\text { ii) } \mathrm{Et}_{3} \mathrm{SiH}}{\stackrel{\mathrm{NMO}}{\longrightarrow}}$ $\mathrm{BF}_{3}$

96a $\mathrm{R}=\mathrm{CO}_{2} \mathrm{Me}$

96b $\mathrm{R}=\mathrm{H}$
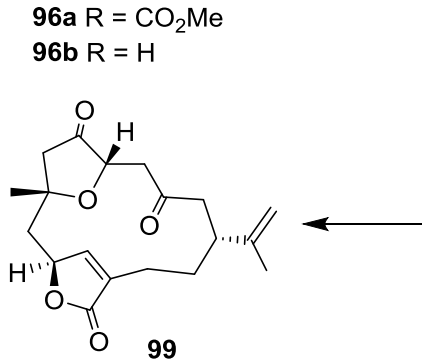

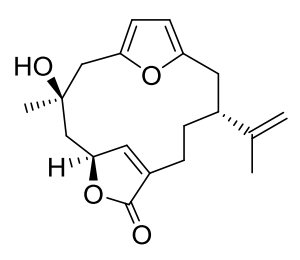

97

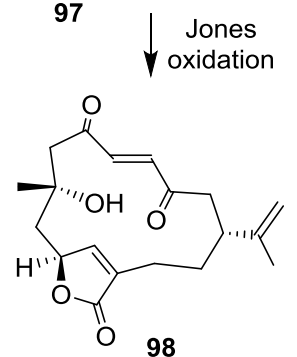

98

Scheme 20. A biogenetically patterned synthesis of norcembrenolide B (99) from norrubifolide $96 \mathrm{~b}$.

have been isolated from Sinularia accommodate a $\mathrm{CO}_{2} \mathrm{Me}$ substituent at $\mathrm{C} 4$ in their structure. Although this biosynthesis proposal, in its entirety, has not been mimicked in the lab, i.e. from for example deoxypukalide $\mathbf{9 6 a}$, inspired by the speculated relationship between FBCs and furanone-based sinuleptolides, Theodorakis et al. ${ }^{[43]}$ synthesised norrubifolide $96 \mathbf{b}, \mathrm{R}=\mathrm{H}$ and showed that after its conversion into the $\mathrm{C} 8$ alcohol furanobutenolide 97 , oxidative cleavage of the furan ring led to the corresponding enedione $\mathbf{9 8}$ which cyclised spontaneously to the furanonebutenolide-based natural product norcembrenolide B (99) (Scheme 20).

Ineleganolide 12 and sinulochmodin C (13) occur with 5episinuleptolide 95 in Sinularia and it has been proposed that the two polycyclic metabolites $\mathbf{1 2}$ and $\mathbf{1 3}$ come from the same furanone-based macrocyle 95 in vivo by successive transannular Michael reactions involving $\mathrm{C} 4-\mathrm{C} 13$ and $\mathrm{C} 7-\mathrm{C} 1$ for

ineleganolide 12 and involving $\mathrm{C} 5-\mathrm{C} 13$ and $\mathrm{C} 7-\mathrm{C} 11$ for sinulochmodin C (13) (Scheme 21). ${ }^{[6]}$

It is likely that scabrolide B (88) is produced in S. scabria by way of a reverse oxy-Michael reaction and cleavage of the ether bridge in the cycloheptanone intermediate (101) to sinulochmodin C (or indeed the latter itself), leading to $\mathbf{1 0 2}$, followed by a transannular Michael reaction (Scheme 22). The biosynthesis of the related metabolites scabrolide A (103) and yonarolide $\mathbf{1 0 4}$ follow from sequential isomerisation of scabrolide $B$ (88) to scabrolide A (103), and dehydration of 103 to yonarolide (104). ${ }^{[6]}$

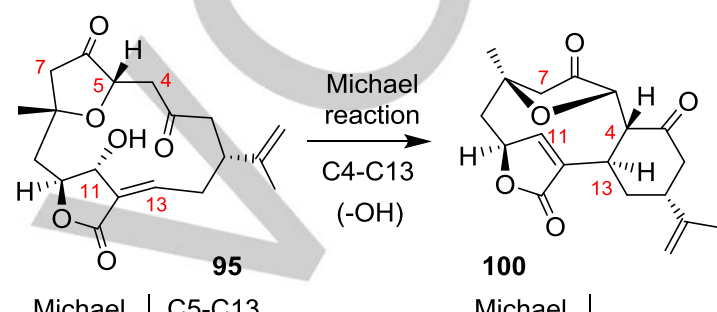

Michael reaction $\downarrow \mathrm{C} 7-\mathrm{C} 11$ reaction $\downarrow(-\mathrm{OH})$

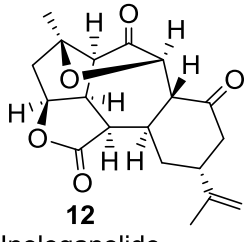

Ineleganolide
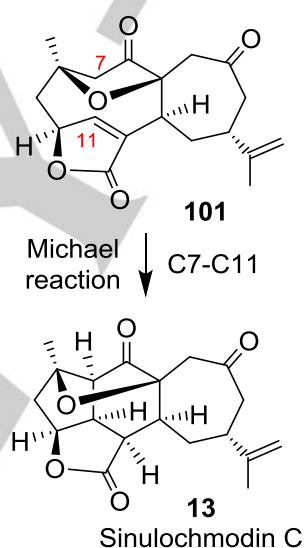

Scheme 21. Proposals for the biosynthesis of ineleganolide 12 and sinulochmodin A (13) from 5-episinuleptolide 95

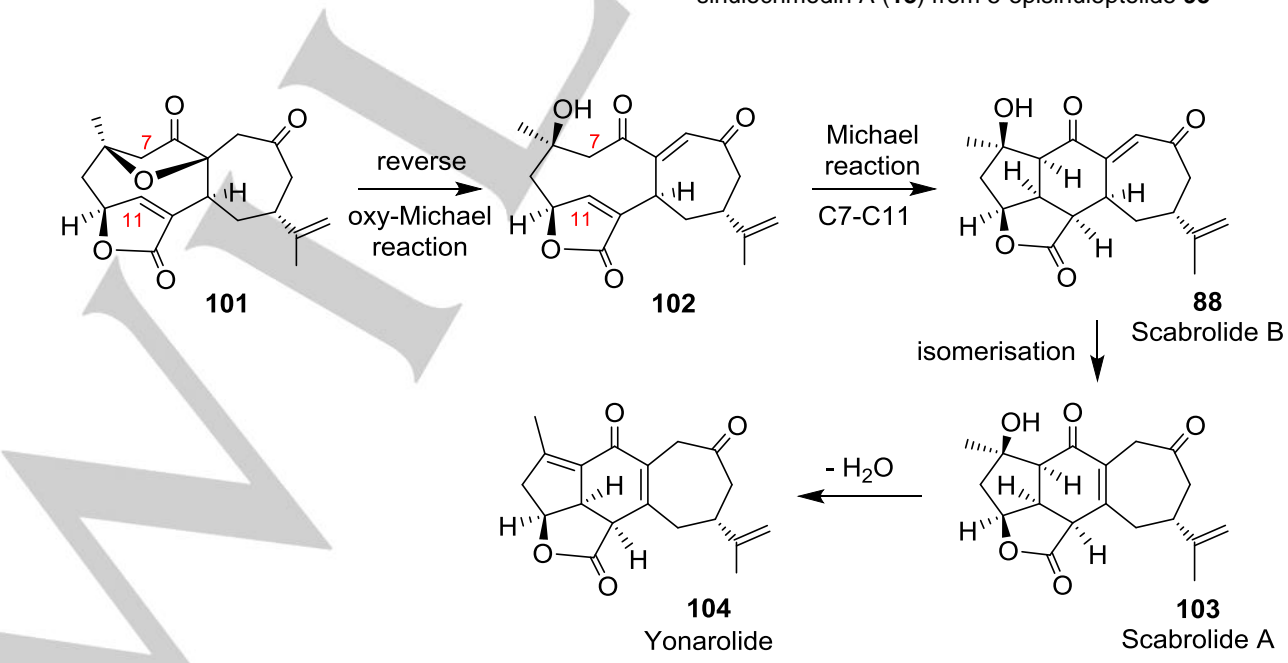

Scheme 22. Proposed biosynthesis of the scabrolides 88 and 103, and of yonarolide 104 
Kavaranolide 89, which was isolated recently alongside ineleganolide 12 from $S$. kavarattiensis, ${ }^{[40]}$ is probably produced in vivo from ineleganolide or its proposed biosynthetic intermediate 100. Thus, a reverse oxy-Michael reaction in ineleganolide 12 or 100 would first lead to $105 \mathrm{~A}$ and 105B respectively. Reverse aldol reactions from 105A and 105B would then give the key trione intermediate 106 (Scheme 23). An intramolecular Michael reaction from 106 would produce horiolide 108, which has been isolated from an Indian ocean collection of Sinularia sp., and an acid-catalysed opening of the butyrolactone ring in $\mathbf{1 0 8}$ would then lead to kavaranolide $\mathbf{8 9}$ (Scheme 23). Interestingly, a competing intramolecular aldol reaction from 106 could produce the novel polycycle 107, which has not yet been reported in Nature (see later discussion).
Several years before the discovery of ineleganolide the unusual metabolite sinulariadiolide $\mathbf{1 1 0}$ had been isolated together with 5-episinuleptolide 95 and yonarolide 104 from an Okinawan Sinularia species. ${ }^{[44]}$ It seems likely that sinulariadiolide is derived from 5-episinuleptolide via oxidative cleavage of the cyclohexanone-based intermediate 109 which would be produced after Michael reaction between $\mathrm{C} 4$ and $\mathrm{C} 13$ in 5episinuleptolide (Scheme 24). ${ }^{[45]}$ It has also been proposed that the novel norcembranoid sinulanocembranolide $A(90)$ found in S. gaweli ${ }^{[41]}$ is also derived from 5 -episinuleptolide 95 by way of a reverse oxy Michael reaction followed by a novel intramolecular dipolar $(3+2)$ cycloaddition, viz 111 $\rightarrow \mathbf{1 1 2}$, as the key step (Scheme 24). ${ }^{[46]}$

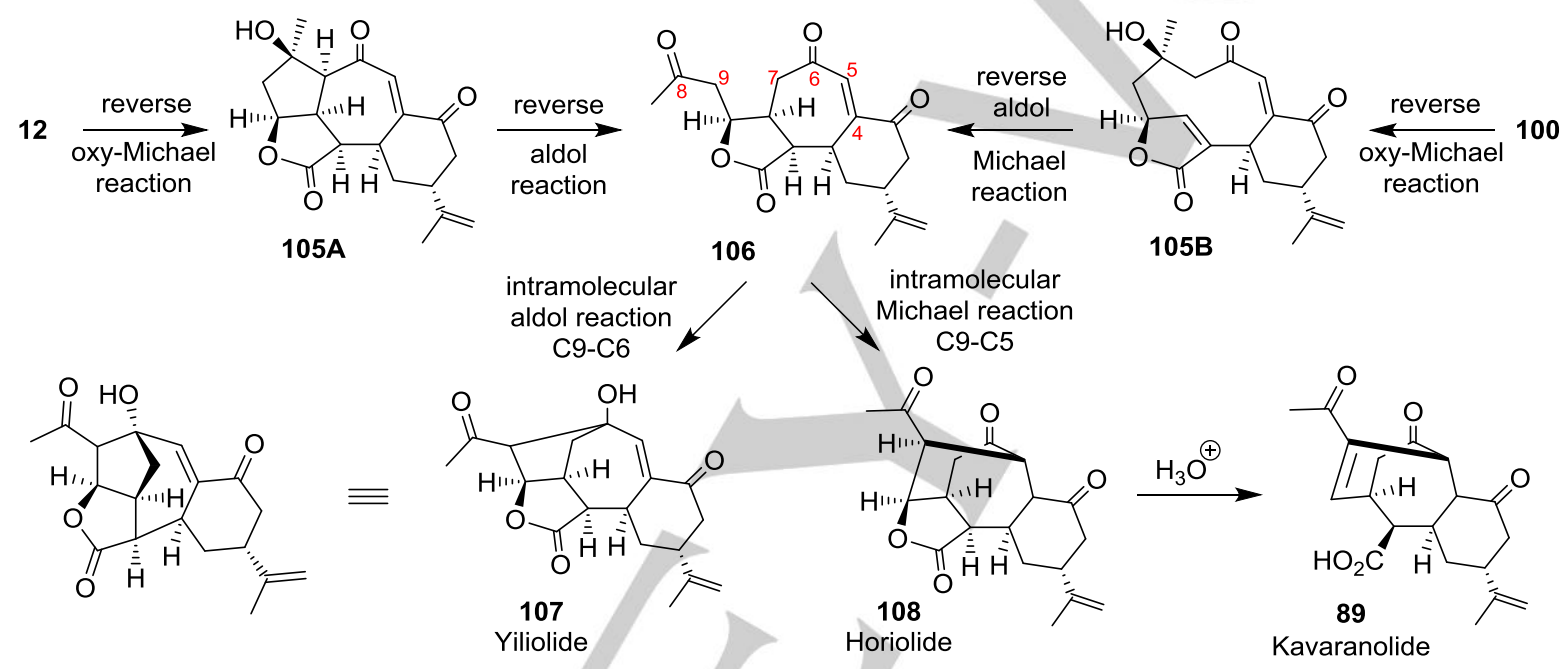

Scheme 23. Proposal for the biosynthesis of kavaranolide 89 and horiolide 108 from ineleganolide 12 or its precursor 100 via the key intermediate 106.

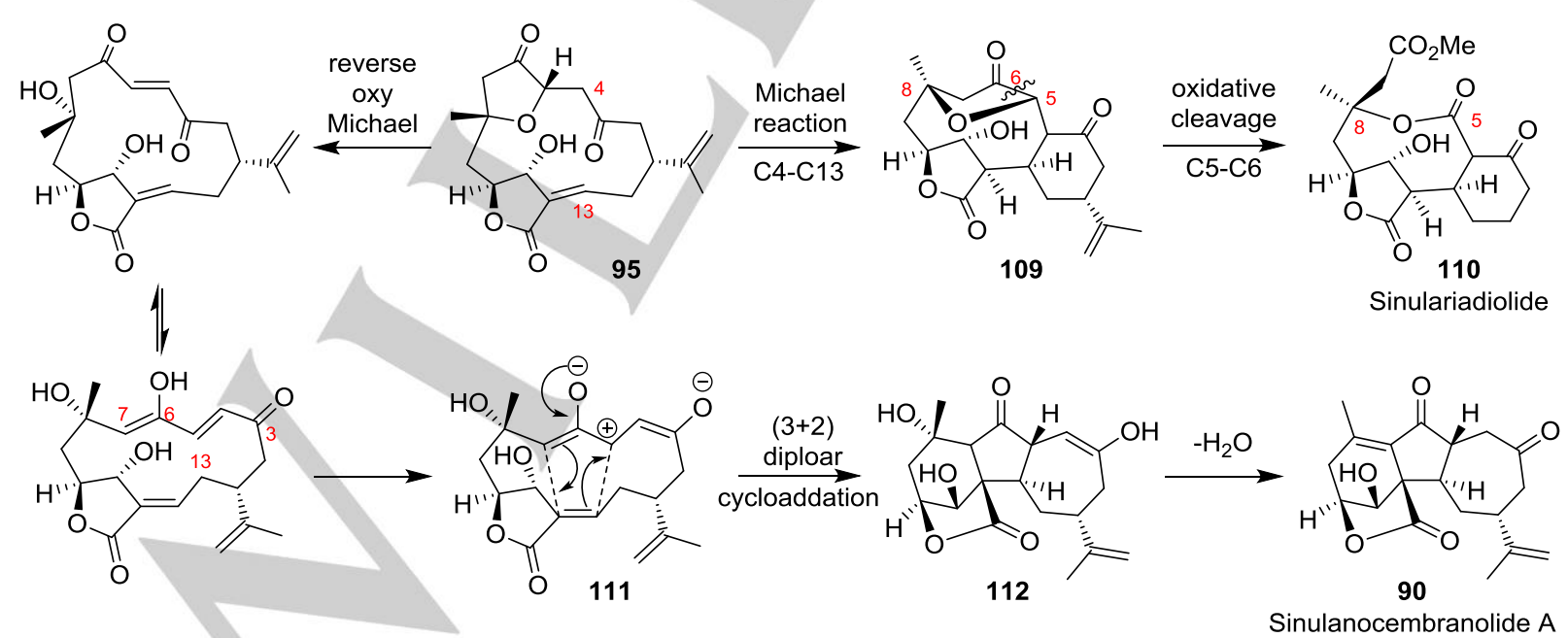

Scheme 24. Proposed origins of sinulariadiolide $\mathbf{1 1 0}$ and sinulnocembranolide $\mathbf{9 0}$ from episinuleptolide $\mathbf{9 5}$ featuring oxidative cleavage, $\mathbf{1 0 9} \rightarrow \mathbf{1 1 0}$, and an intramolecular dipolar [3+2] cycloaddition, viz 111 $\rightarrow \mathbf{1 1 2}$. 
An interesting biosynthetic route to dissectolide 14, also from 5episinuleptolide 95, implicating either an intramolecular (4+2) cycloaddition from the diene intermediate $\mathbf{1 1 3}$ or a Michael cyclisation / vinylogous aldol process, $\mathbf{1 1 3} \rightarrow \mathbf{1 1 4} \rightarrow \mathbf{1 1 5}$, has been put forward (Scheme 25).

Sinugyrosanolide A (117) is yet another interesting 5,7,6 ringfused norcembranoid which was isolated from the Formosan soft coral S. gyrosa in 2014. ${ }^{[47]}$ This metabolite is also likely to be derived from 5-episinuleptolide 95 following a reverse oxy Michael reaction and concomitant 1,4-elimination between $\mathrm{C} 11$ and C14 in 95 leading to the intermediate 116 (Scheme 26). The macrocyclic trienedione $\mathbf{1 1 6}$ is nicely predisposed to take part in an intramolecular $(4+3)$ type cycloaddition reaction leading directly to the natural product. The $(4+3)$ cycloaddition $\mathbf{1 1 6} \rightarrow \mathbf{1 1 7}$ is similar to one of the proposals put forward for rameswaralide 10 discussed earlier. Sinugyrosanolide A (117) corresponds to C4 descarboxyrameswaralide and the authors who isolated the natural product have suggested that it might be derived in vivo from rameswaralide; the same authors have also put forward a more circuitous biosynthetic proposal to $\mathbf{1 1 7}$ from scabrolide $F$ (118).

Over the past decade a number of chemists have made huge efforts to achieve a total synthesis of the highly oxygenated polycyclic norcembranoid ineleganolide 12. Disappointingly, all of these synthetic efforts, although getting very close to the target, have so far been unsuccessful. ${ }^{[14]}$

In 2011, very soon after its structure was published, Pattenden et al. rationalised the biosynthesis of ineleganolide 12 occurring from 5-episinuleptolide $95 .{ }^{[6]}$ Thus, we suggested that the 5,7,6 angular tricyclic ring system in ineleganolide was produced from the fourteen-membered ring system in 5-episinuleptolide 95 by way of successive transannular Michael reactions between the $\mathrm{C} 4, \mathrm{C} 13$ and $\mathrm{C} 7, \mathrm{C} 11$ centres in its structure. Furthermore, since sinulochmodin C (13) co-occurs with ineleganolide and 5episinuleptolide in Sinularia it seemed probable that the corresponding 5,6,7 linear tricyclic ring system in sinulochmodin C (13) was also derived from 5-episinuleptolide by an alternative sequence of transannular Michael reactions involving the C5,C13 and C7,C11 centres in the latter. These proposals were set out earlier in Scheme 21. With a view to vindicating our proposals we obtained a small sample of natural 5episinuleptolide which had been isolated from Sinularia sp, through the generosity of Professor $\mathrm{J}-\mathrm{H}$. Sheu, National Sun Yat-sen University, Taiwan). In the lab we found that 5episinuleptolide 95 remained unchanged on treatment with bases and hence we prepared the corresponding acetate 119

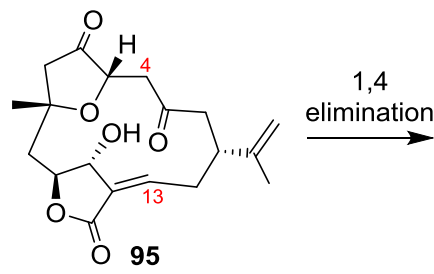

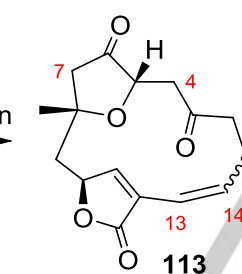

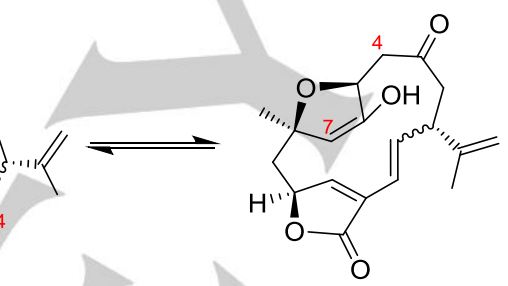

Michael reaction
5 -exo-trig

[4+2]

cycloaddition
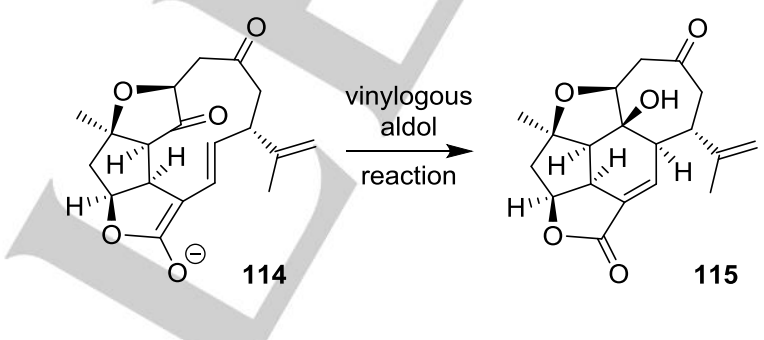

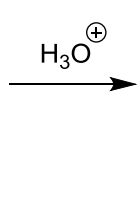

115

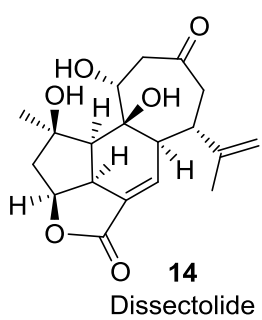

Scheme 25. Proposal for the origin of dissectolide 14 from 5-episinuleptolide 95 implicating the vinybutenolide intermediate 113.
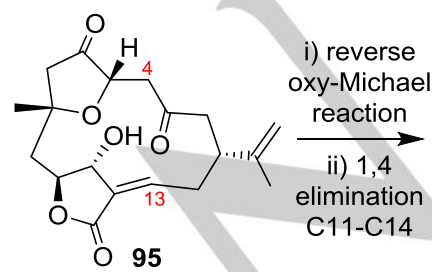

C11-C14

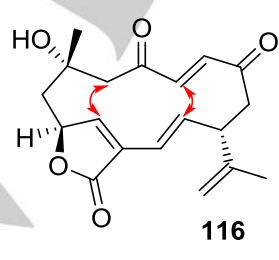

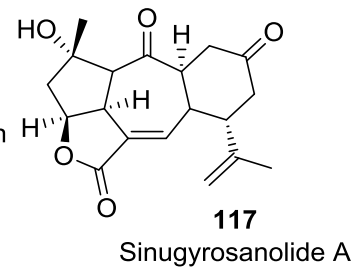

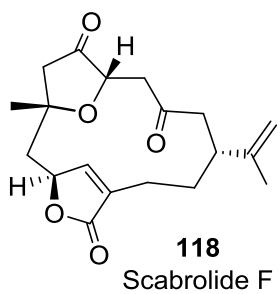

Scheme 26. Proposals for the biosynthesis of sinugyrosanolide A (11) from 5-episinuleptolide 95 


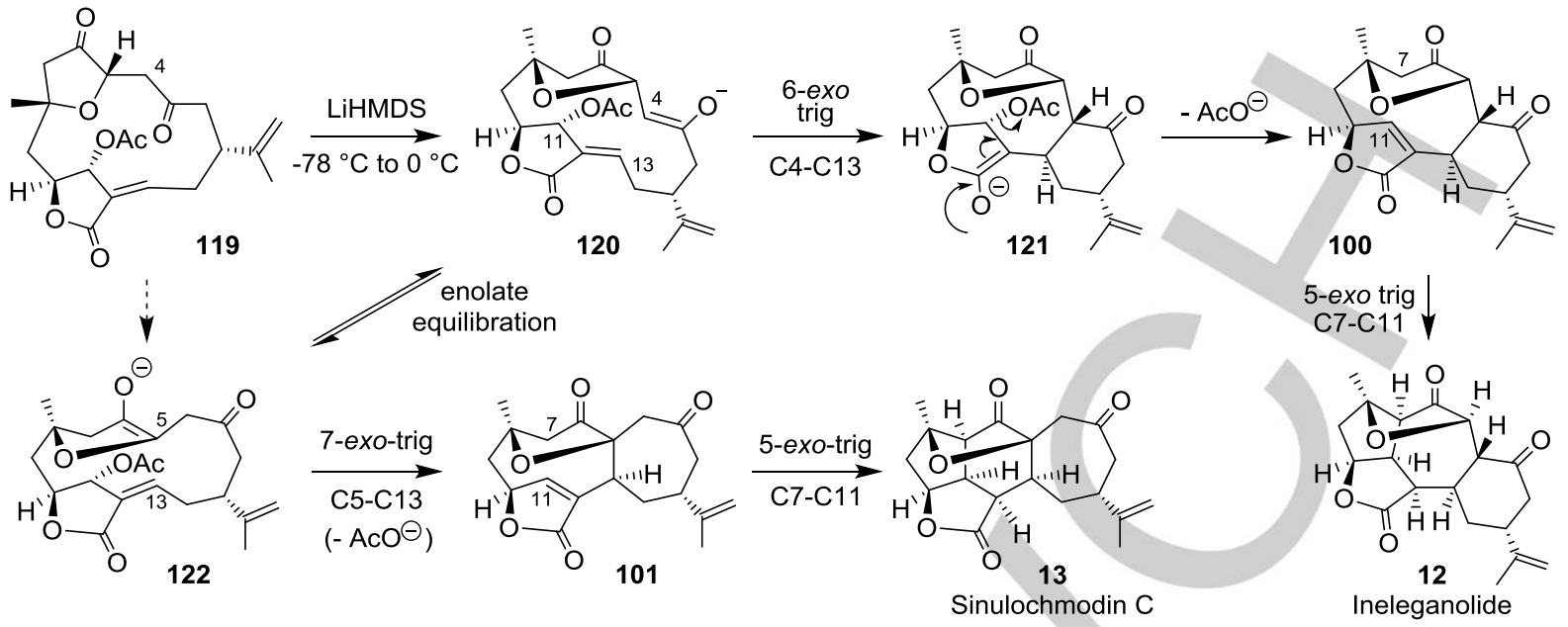

Scheme 27. Biomimetic synthesis of ineleganolide 12 and sinulochmodin C(13) from 5-episinuleptolide acetate 119 in the presence of lithium HMDS.

To our satisfaction when a solution of the acetate 119 in THF was treated with lithium hexamethydisilazide (LiHMDS) at $-78^{\circ} \mathrm{C}$ to $0{ }^{\circ} \mathrm{C}$, work-up gave a mixture of ineleganolide 12 and sinulochmodin C (13) (ca. $80 \%$ crude) which was cleanly separated by chromatography. ${ }^{[48]}$ The straightforward one-pot conversion of 5-episinuleptolide into ineleganolide and sinulochmodin $C$ in the presence of LiHMDS seems quite remarkable and provided convincing support for the biosynthesis proposals we made earlier to these novel structures. Interestingly, several months after this work was published, Yen et al. ${ }^{[49]}$ isolated 5 -episinuleptolide acetate 119 from a soft coral Sinularia sp collected off the coast of Taiwan.

It seems likely that, as proposed, the angular 5,7,6-ring fused system in ineleganolide $\mathbf{1 2}$ is produced by initial deprotonation of the more sterically accessible C4 centre in the acetate 119 leading to the enolate $\mathbf{1 2 0}$ which then undergoes 6-exo trig cyclisation producing intermediate 121 (Scheme 27). Displacement of the acetate in $\mathbf{1 2 1}$ next leads to $\mathbf{1 0 0}$ which undergoes deprotonation at $\mathrm{C} 7$ followed by 5 -exo trig cyclisation providing ineleganolide 12. The isomeric linear 5,6,7-ring fused system in sinulochmodin C (13) most likely arises from the enolate 122 produced from equilibration with 120 [or directly from the acetate 119] by 7-exo trig cyclisation between $C 5$ and C13 accompanied by displacement of the OAc group at C11 leading to 101 followed by 5 -exo trig cyclisation between $\mathrm{C} 7$ and C11 in the latter (Scheme 27).

Intriguingly, when 5-episinuleptolide acetate 123 was treated with sodium instead of lithium HMDS at higher temperature, i.e. $40{ }^{\circ} \mathrm{C}$ to $-10{ }^{\circ} \mathrm{C}$ a clean reaction gave the new polycyclic norcembranoid 107 in $75 \%$ yield. ${ }^{[48]}$ The new structure 107 , which we have named yiliolide, has not yet been reported as a natural product but it clearly has features in common with horiolide 108 and kavaranolide 89 found in Sinularia. As we have discussed earlier in Scheme 23 we suggest that similar to its isomers 108 and $\mathbf{8 9}$, yiliolide is produced from ineleganolide by way of a competitive aldolisation reaction from the key intermediate 106 which leads to horiolide and kavaranolide in vivo.

It is interesting that the biosynthetic speculations presented above, leading to the formation of polycyclic norcembranoids in soft corals of the genus Sinularia, all start from the same $3(2 H)$ furanone ring-based macrocycle 5-episinuleptolide 95. This metabolite was first reported in 1978 but since then a number of related furanone structures with different oxidation patterns between $\mathrm{C} 11-\mathrm{C} 13$ and stereochemistry at $\mathrm{C} 5$ and $\mathrm{C} 8$ have been described. ${ }^{[6]}$

More interestingly, Sheu et al. ${ }^{[50]}$ have isolated the C20 isocembranoid 124 and the episinuleptolide dimer 123 alongside 5-episinuleptolide 95 and sinulochmodin $C$ (13) from $S$. lochmodes (Scheme 28) which has raised the question whether or not carbon centre radicals, e.g the furanone radical 125 rather than the enolate 126 (cf. 122 in Scheme 27) might be involved in the biosynthesis of sinulochmodin $\mathrm{C}$ and, indeed, other polycyclic norcembranoids. ${ }^{[6]}$

Carbon centred radicals are often suggested as intermediates in biosynthetic pathways and can be produced in vivo in a variety of ways, including photochemical excitation, electron transfer and oxidation reactions. The particular captodative radical $\mathbf{1 2 5}$ is relatively stable and it is not surprising that it dimerises to $\mathbf{1 2 3}$ in vivo. However, efforts to synthesise sinulochmodin C (13) via 125 from treatment of 5-episinuleptolide 95 with a number of radical-initiating conditions have not been successful. ${ }^{[46]}$ Albeit interesting, treatment of $\mathbf{9 5}$ with samarium diiodide instead led to the 6,7,5-ring fused polycyclic structure 127 in $62 \%$ yield (Scheme 29) instead of to sinulochmodin $C(13) .{ }^{[46]}$ The jury remains out therefore as to whether radical intermediates might be involved in any of the biosynthetic sequences presented here leading to polycyclic norcembranoids from episinuleptolide. 


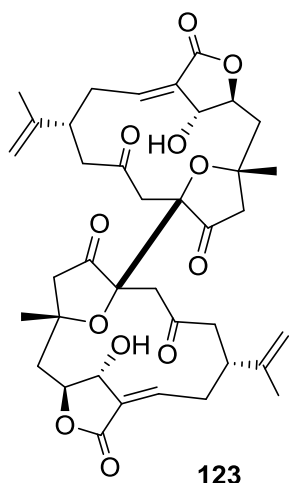

123

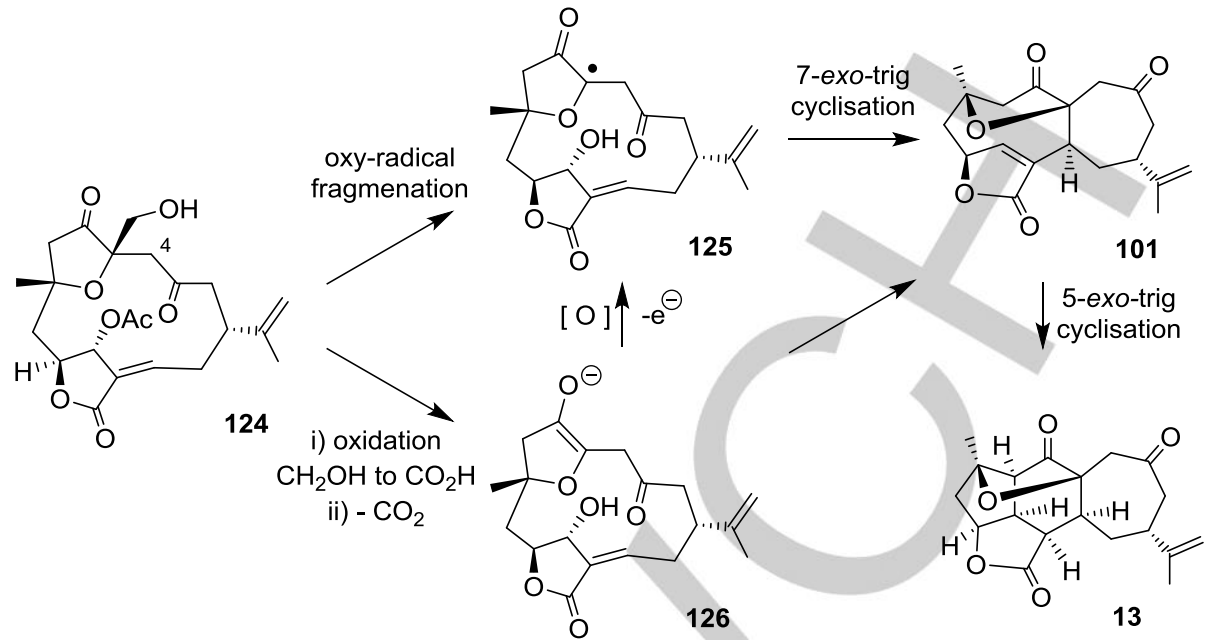

Scheme 28. Proposal for the biosythesis of sinulochmodin C (13) implicating the radical intermedate 125.

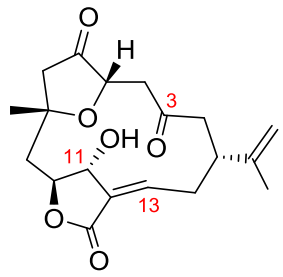

95

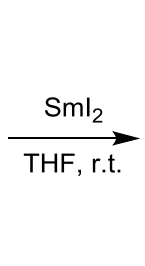

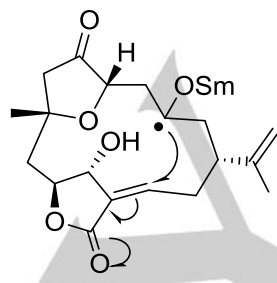
O)

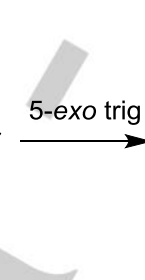

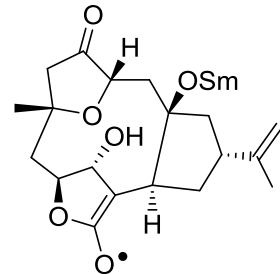
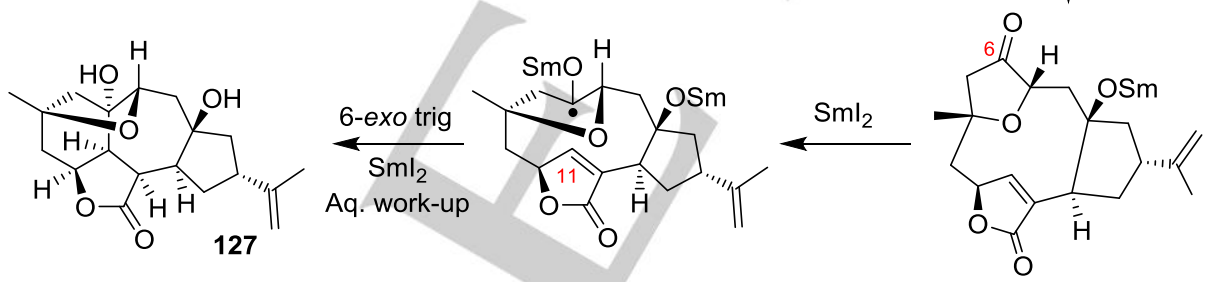

Scheme 29. Proposal for the origin of the polycycle 127 resulting from treatment of 5 -episinuleptolide 95 with samarium diiodide in THF.

\section{Other cembranoid-derived secondary metabolites}

Pseudopteranes (also known as kallolides) are macrocyclic 12membered ring furanobutenolide-based cembranoids which were first isolated from Pseudopterogorgia sp during the 1980s. ${ }^{[51,52]}$ In 1998 Rodrigues et al. ${ }^{[53]}$ isolated kallolide A (128) alongside bipinnatin $\mathrm{J}(3)$ from $P$. bipinnata and found that when they irradiated a solution of bipinnatin $J$ in acetonitrile with a medium pressure $\mathrm{Hg}$ lamp through Pyrex it underwent facile ring contraction to kallolide A (Scheme 30). This single photochemical (biomimetic) reaction, for the first time, established the biogenetic relationship between a 14-membered furanobutenolide-based cembranoid and its 12-membered ring isomer.

More recently, following the characterisation of $Z$-and $E$-isomers, 130 and 131 respectively, of deoxypukalide in the Pacific coral
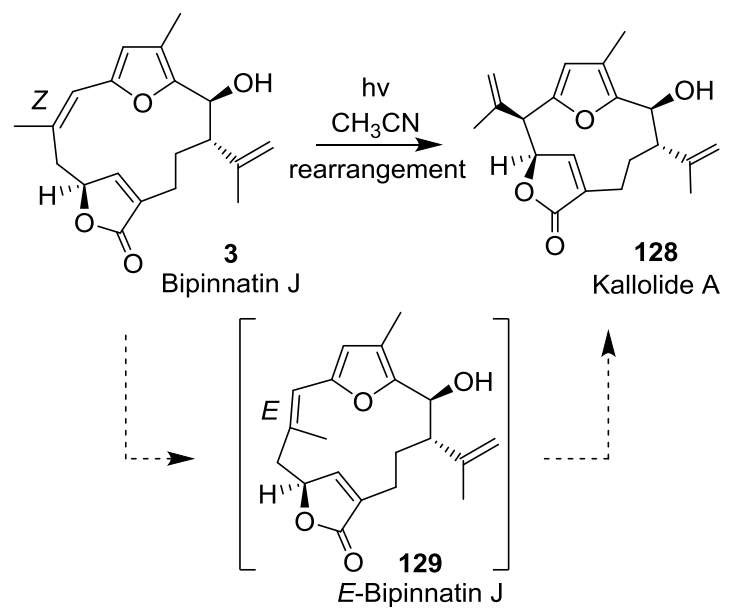

Scheme 30. Photochemical ring contraction of bipinnatin $\mathrm{J}(\mathbf{3})$ to the 12 membered ring-containing kallolide A (128). 
Leptogorgia Pattenden et al. ${ }^{[54]}$ examined the photochemical behaviour of (Z)-bipinnatin $\mathrm{J}(\mathbf{3})$ in more detail alongside that of naturally occurring Z-deoxypukalide $\mathbf{1 3 0}$ and Z-rubifolide 1. In each study it was found that short irradiation times resulted only in isomerisations of the $Z$ - alkene bonds in 3, 130 and 1 leadingto the corresponding $E$-isomers 129, 131 and 133 respectively. However, when the same irradiations were continued for longer periods of time the anticipated ring contractions occurred from the intermediate $E$-isomers leading to kallolide A (128), deoxypseudopterolide 132 and kallolide $B$ (7) respectively (Scheme 31). Deoxypseudopterolide 132 was first described alongside acerosolide 5 in Pseudopterogorgia corals during the 1980s. The closely related 11-gorgiacerol 135 and its $\mathrm{C} 11$ epimer were later found in the coral $P$. acerosa,<smiles>[Z20]O[R6]([H])([H])O[Na]</smiles>

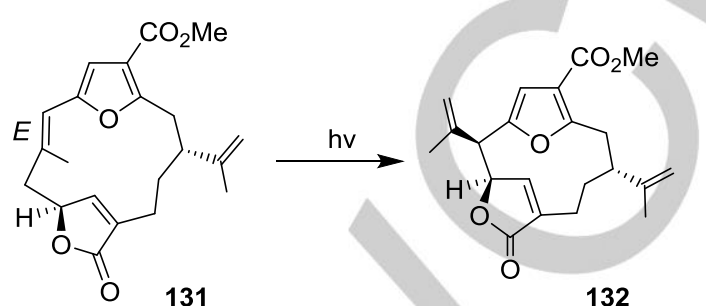

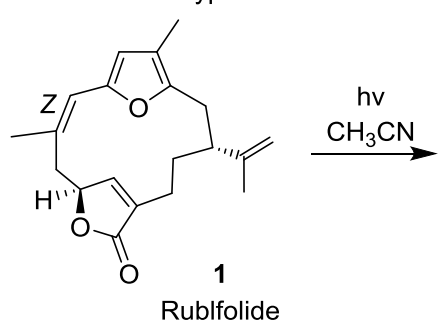

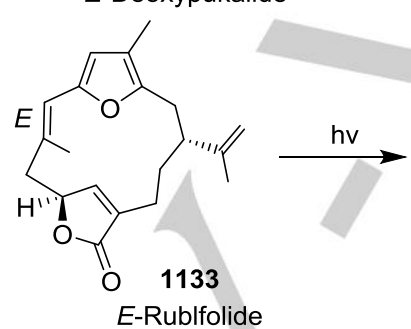

Deoxypseudopterolide

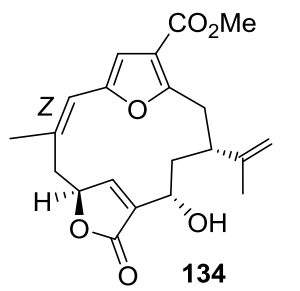

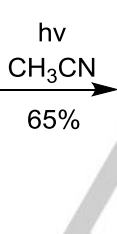

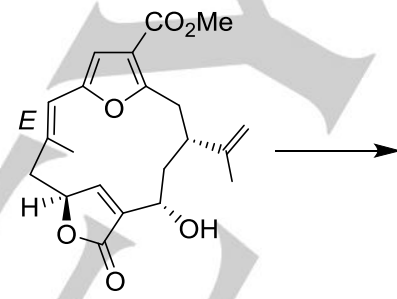

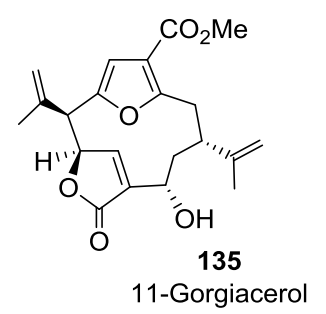

Scheme 31. Photochemical $Z \rightarrow E$ isomerisations of $Z$ - FBCs followed by $14 \rightarrow 12$-membered ring contractions leading to pseudopteranes and kallolides..

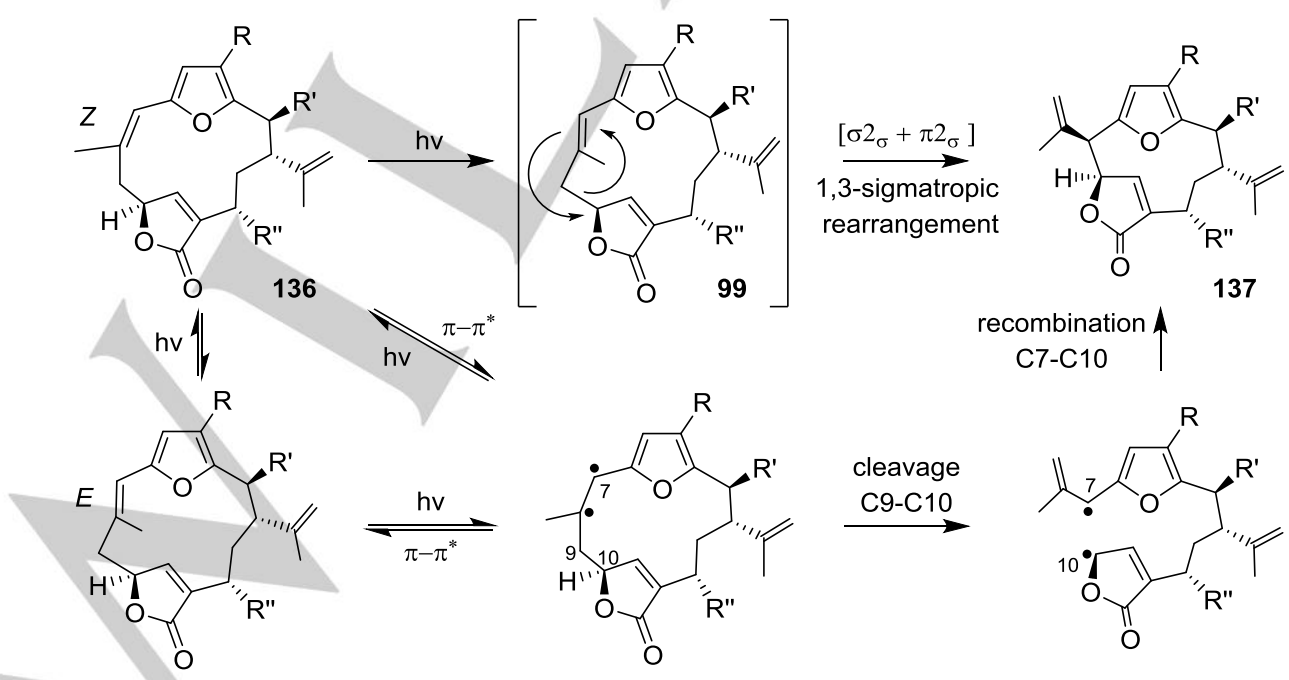

Scheme 32. Proposed 1,3-sigmatropic rearrangement and radical mechanisms for the photo-induced ring contractions of FBCs to pseudopteranes/kallolides. 
and Mulzer et al. ${ }^{[5]}$ have described a biomimetic synthesis of 135 and also its $\mathrm{C} 11$ epimer following irradiation and ring contraction of the Z-configured C11 epimeric FBC 134.

The $14 \rightarrow 12-$ membered ring contractions shown in Schemes 30 and 31 are stereospecific 1,3-sigmatropic rearrangements, i.e. $\mathbf{1 3 6} \rightarrow \mathbf{1 3 7}$ (Scheme 32), which are allowed photochemically. The isomerisations of the Z-FBCs to the corresponding $E$-isomers, which seem necessary prior to ring contraction, are initiated by $\pi-\pi^{*}$ excitation of the $C 7, C 8$ alkene bonds in 136. It is also possible therefore to represent the same $14 \rightarrow 12$-membered ring contractions proceeding via the radical pathway shown in Scheme 32. ${ }^{[56]}$

Interestingly, Rodrigues et $a l^{[53]}$ also found the novel cyclopropane ring-containing pinnatin A $(\mathbf{1 3 8}) \quad(<0.5 \%$ yield $)$ alongside kallolide A (129) after irradiation of bipinnatin $\mathrm{J}(\mathbf{3})$ through quartz instead of Pyrex. Furthermore, pinnatin $A$ has been found with kallolide $A$ and bipinnatin $J$ in P.bipinnata. The "biomimetic" isomerisation of bipinnatin $\mathrm{J}(3)$ into pinnatin A (138) is accompanied by complete epimerisation of the $\mathrm{OH}$

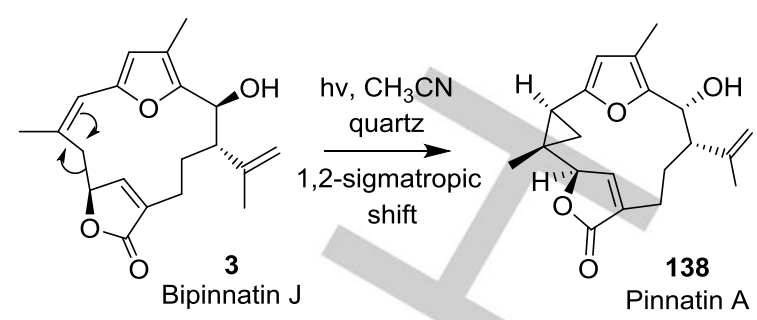

Scheme 33. Photochemical 1,2-sigmatropic rearrangement, accompanied by epimerisation at $\mathrm{C} 2$, of bipinnatin $\mathrm{J}(\mathbf{3})$ leading to pinnatin $\mathrm{A}(\mathbf{1 3 8})$.

group at $\mathrm{C} 2$, and has been represented as a $\left[\alpha 2_{s}+\pi 2_{s}\right]$ cycloaddition reaction (Scheme 33).

Coralloidolides B (140) and C (142), found in the Mediterranean coral Alcyonium coralloides, are examples of another family of natural products whose biosynthesis Trauner $^{[57]}$ and others ${ }^{[5]}$ proposed to be from the FBC rubifolide 1 by oxidation and oxidative cleavage to the epoxy enedione 139 (coralloidolide E)

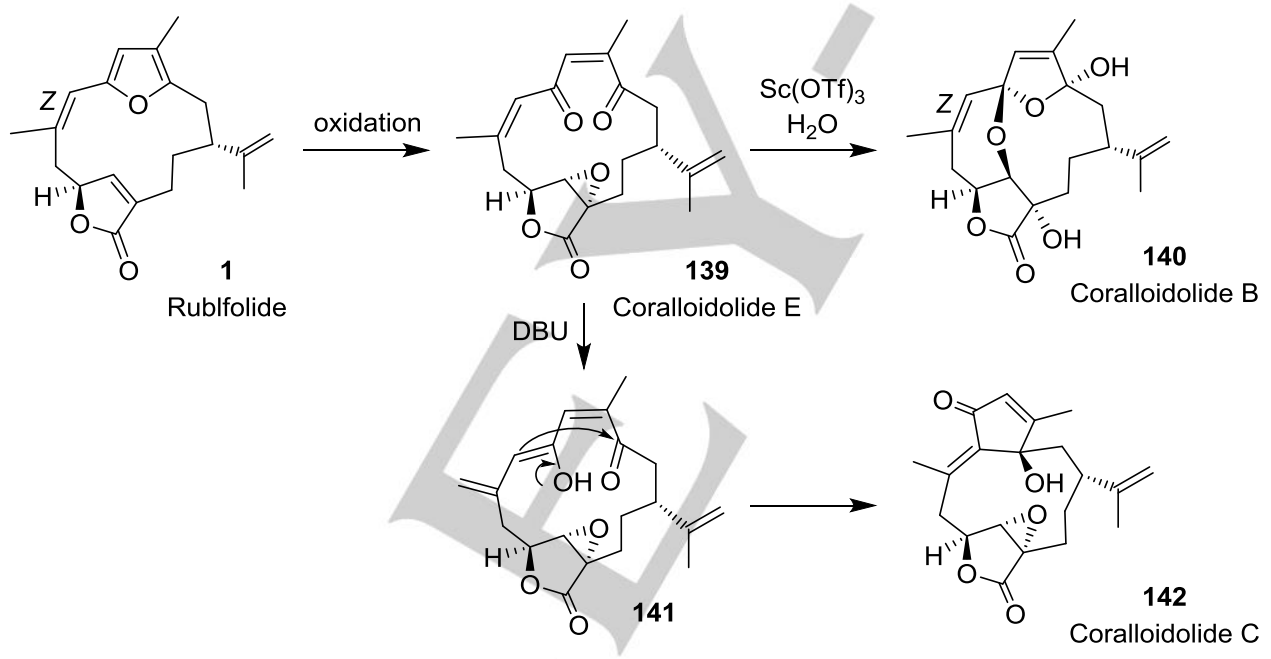

Scheme 34. Biomimetic syntheses of coralloidolides from rubifolide 1 following oxidation cleavage and skeletal rearrangement.
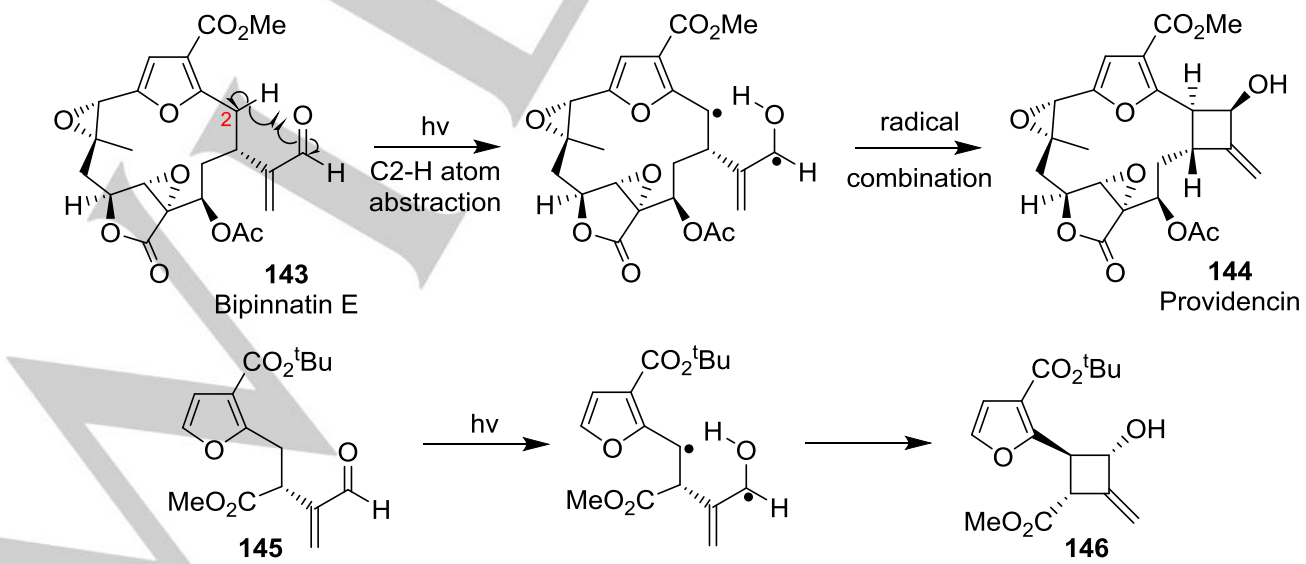

Scheme 35. A proposed C-H insertion reaction leading to the methylenecyclobutanol unit in the biosynthesis of providencin $\mathbf{1 4 4}$ from bipinnatin $\mathrm{E}$ (143) 
followed by skeletal rearrangement. Trauner and his group later synthesised coralloidolide E (135) from rubifolide 1 which, after extensive experimentation, they were able to convert into coralloidolide B (140) on treatment with hydrated scandium triflate (Scheme 34$).^{[57]}$ In other biomimetic experiments, treatment of 139 with DBU was shown to lead to coralloidolide C (142) presumably via an intramolecular aldol reaction from the enol intermediate 141.

This Perspective would not be complete without a brief mention of the unique cyclobutane-ring containing metabolite providencin 144 isolated from $P$. kallos, if only to acknowledge the considerable amount of effort that has been committed to its synthesis in the lab. over the past decade. ${ }^{[58]}$ The biosynthesis of the methyleneyclobutanol ring in providencin is most likely produced in vivo from bipinnatin $E$ (143) by a photochemical route implicating an intramolecular insertion reaction (NorrishYang type) of the $\mathrm{CHO}$ functionality at $\mathrm{C} 16$ into the $\mathrm{C}-\mathrm{H}$ bond at $\mathrm{C} 2$ in 143 (Scheme 35). ${ }^{[59]}$ Indeed this novel C-H insertion reaction, which was unprecedented, has been modelled successfully using the furan substrate $\mathbf{1 4 5}$ which on irradiation was shown to lead to the substituted methylenecyclobutanol 146 in acceptable yield. ${ }^{[59]}$

An alternative proposal to the cyclobutane ring in providencin implicating initial cyclisation of a carbocation at C2 into a propenyl residue at $\mathrm{C} 1$ followed by further specific oxidation seems less attractive. ${ }^{[58 a]}$ Nevertheless, a non-biomimetic route has been developed to 17-deoxyprovidencin which allows at least the final part of this alternative biomimetic proposal to be evaluated. In addition, Tang and Paton, ${ }^{[60]}$ have recently used DFT calculations to assess the $\mathrm{C}-\mathrm{H}$ insertion proposal to providencin and they show that not only is the cyclisation possible but the stereochemical outcome of the cyclisation is consistent with that found in providencin.

\section{Concluding remarks}

In the absence of any biosynthesis studies, speculations on the likely origins of secondary metabolites will always be considered by some as "an interesting paper exercise". Nevertheless, the speculations presented in this personal Perspective, have led to plausible biomimetic syntheses of the marine natural products intricarene 11, ineleganolide 12 and sinulochmodin $C$ (13), in particular, and also to the pseudopteranes / kallolides, e.g. 7 and coralloidolides, e.g. 137. In addition, other biomimetic studies have provided a wealth of new synthetic and mechanistic knowledge particularly on the complex pathways to the ring systems in beilschowskysin 8 , plumarellide 9 and rameswaralide 10 involving novel furanoxonium ions as key intermediates.

It goes without saying that the isolation of similar secondary metabolites from the same organism does not always signal that one is the actual precursor of another. Indeed there is evidence that some metabolites isolated from corals are actually biosynthesised by the symbiotic microorganisms that the coral frequently shelters. There is also the issue that some of the compounds isolated from corals are not natural products at all, but in fact artefacts of the isolation procedures formed during work up, i.e by oxidation and /or photochemical processes.

A range of oxidation processes have been invoked in the speculated biosynthetic pathways to polycyclic cembranoids from FBCs in corals described here, and although we know little about the enzymes that are involved those based on P450 monoxygenases are surely implicated. Other oxidations triggered by light are also rife. The photon is also the "reagent" in a number of biosynthetic $\mathrm{C}-\mathrm{C}$ bond forming reactions and rearrangements, and it is not possible to avoid implicating radical intermediates in many of these processes, $c f$. intricarene and the pseudopteranes.

An arsenal of common ionic reactions, particularly Michael reactions and aldol processes (and their reverse processes), and cycloaddition (including dipolar) reactions are implicated in the speculations presented in this Perspective. Indeed the subtle balance of competing aldol and Michael reactions which lead from 5-episinuleptolide 95 to ineleganolide 12, sinulochmodin C (13) and yiliolide (107) is really quite mind-boggling !

Similar to designing a synthetic route to a complex natural product structure, there is nothing more satisfying than speculating on the likely biosynthesis of the same complex structure and then vindicating your proposal in the lab. You feel you have not only accomplished a challenging target but that you have also uncovered some of Nature's secrets.

This will probably be the final contribution that Gerry Pattenden will make to this area, and I hope that the beauty and complexity of the structures of polycyclic cembranoids found in corals, alongside the variety of biological properties they display, will continue to entice and challenge the next generation of chemists to further explore their total synthesis and biomimetic synthesis for several years to come.

\section{Acknowledgments}

We are greatly indebted to the ever-diminishing number of creative natural product isolation scientists who have delivered a wide variety of intriguing structures over the past decades to excite and challenge the imaginations of we mere synthetic chemists.

We also thank the School of Chemistry at The University of Nottingham for support and encouragement, and for providing access to facilities.

Keywords: Biomimetic synthesis - Cycloaddition - Natural products $\cdot$ Macrocycles $\cdot$ Furanobutenolide-cembranoids

\section{References and notes}

[1] Y. Li, G. Pattenden, Nat. Prod. Rep. 2011, 28, 1269-1310.

[2] P. A. Roethle, D. Trauner, Nat. Prod. Rep. 2008, 25, 298-317.

[3] a) For annunal reviews on marine natural products, see: J. W. Blunt, A R. Carroll, B. R. Copp, A. Davis, R. A. Keyzers, M. R. Prinsep, Nat. Prod. Rep. 2018, 35, 8-53 and all preceding reviews in this series; b) F. 
Berrue, R. G. Kerr, Nat. Prod. Rep. 2009, 26, 681-710; c) J. Marrero, I. I. Rodríguez, A. D. Rodríguez, Comprehensive Natural Products II, Chemistry and Biology; L. Mander, H.-W. Liu, Eds.; Elsevier: Oxford, 2010, 2; 363-428.

[4] a) B. Yang, X.-F. Zhou, X.-P. Lin, J. Liu, Y. Peng, X.-W. Yang, Y. Liu, Curr. Org. Chem. 2012, 16, 1512-1539; b) L. C. A. Barbosa, J. O. S Varejao, E. V. V. Vajeyao, Studies in Natural Product Chemistry, 2017, 52, 115-157.

[5] a) M. D'Ambrosio, D. Fabbri, A. Guerriero, F. Pietra, Helv. Chim. Acta, 1989, 70, 63; b) M. D’Ambrosio, A. Guerriero, F. Pietra, Helv. Chim. Acta, 1990, 73, 804-807.

[6] For pertinent literature see; Y. Li, G. Pattenden, Nat. Prod. Rep. 2011, 28, 429-440.

[7] J. Marrero, A. D. Rodríguez, P. Baran, R. G. Raptis, J. A. Sanchez, E. Ortega-Barria, T. L. Capson, Org. Lett. 2004, 6, 1661-1664.

[8] V. A. Stonik, I. I. Kapustina, A. I. Kalinovsky, P. S. Dmitrenok, B. B. Grebnev, Tetrahedron Lett. 2002, 43, 315-317

[9] P. Ramesh, N. S. Reddy, Y. Venkateswarlu, M. V. R. Reddy, D. J. Faulkner, Tetrahedron Lett. 1998, 39, 8217-8220.

[10] J. Marrero, A. D. Rodríguez, C. L. Barnes, Org. Lett. 2005, 7, 1877-1880.

[11] C.-Y. Duh, S.-K. Wang, M.-C. Chia, M. Y. Chiang, Tetrahedron Lett. 1999, 40, 6033-6035.

[12] Y.-J. Tseng, A. F. Ahmed, C.-F. Dai, M. Y. Chiang, J.-H. Sheu, Org. Lett. 2005, 7, 3813-3816.

[13] M. Kobayashi, K. M. C. A. Rao, M. M. Krishna, V. Anjaneyulu, J. Chem. Res. (S) 1995, 188-189.

[14] R. A. Craig, B. M. Stoltz, Chem. Rev. 2017, 117, 7878-7909.

[15] A. D. Rodríguez, Y.-P. Shi, J. Org. Chem. 2000, 65, 5839-5842

[16] A. Saitman, E. A. Theodorakis, Org. Lett. 2013, 15, 2410-2413.

[17] Y. Li, G. Pattenden, J. Rogers, Tetrahedron Lett. 2010, 51, 1280-1283.

[18] K. C. Nicolaou, V. A. Adsool, C. R. H. Hale, Angew. Chem., Int. Ed. 2011, 50, 5149-5152.

[19] S. D. Townsend, G. A. Sulikowski, Org. Lett. 2013, 15, 5096-5098.

[20] B. Tang, R. Simion, R. S. Paton, Synlett, 2015, 26, 501-507.

[21] P. Scesa, M. Wangpaichitr, N. Savaraj, L. West, S. P. Roche, Angew. Chem., Int. Ed., 2018, 57, 1316-1321.

[22] Y. Venkateswarlu, M. A. F. Biabani, M. V. R. Reddy, T. P., Roa, A. C. Kunwar, D. J. Faulkner, Tetrahedron Lett. 1994, 35, 2249-2252.

[23] A. S. R. Anjaneyulu, K. S. Sagar, M. J. R. V. Venugopal, Tetrahedron 1995, 51, 10997-11010.

[24] B. R. Chitturi, V. B. Tatipamula, C. B. Dokuburra, U. K. Mangamuri, V. R. Tuniki, S. V. Kalivendi, R. A. Bunce, V. Yenamandra, Tetrahedron, 2016, 72, 1933-1940.

[25] S. A. L. Thomas, J. L. von Salm, S. Clark, S. Ferlita, P. Nemani, A. Azhari, C. A. Rice, N. G. Wilson, D. E. Kyle, B. J. Baker, J. Nat. Prod 2018, 81, 117-123.

[26] G. Pattenden, J. M. Winne, Tetrahedron Lett. 2009, 50, 7310-7313.

[27] M. J. Palframan, G. Pattenden, Tetrahedron Lett. 2013, 54, 324-328.

[28] Y. Li, M. J. Palframan, G. Pattenden, J. M. Winne, Tetrahedron 2014, 70, 7229-7240.

[29] a) J. M. Winne, S. Catak, M. Waroquier, V. V. Speybroeck, Angew. Chem., Int. Ed., 2011, 50, 11990-11993; b) J. Hullaert, J. M. Winne, Angew. Chem. Int. Ed. 2016, 55, 13254-13258; c) J. Hullaert, B. Denoo M. Christiaens, B. Callebaut, J. M. Winne, Synlett, 2017, 28, 2345-2352.

[30] see: M. J. Palframan, G. Pattenden, Chem Commum. 2014, 50 , 7223-7242.

[31] M. J. Palframan, G. Pattenden, Synlett. 2013, 24, 2720-2722.

[32] a) B. Lygo, M. J. Palframan, G. Pattenden, Org. Biomol. Chem. 2014 12, 7270-7278; b) see also reference 29 a
[33] Y. Li, G. Pattenden, Tetrahedron Lett. 2011, 52, 2088-2092.

[34] A. S. Anjaneyulu, M. J. Venugopal, P. Sarada, J. Clardy, E. Lobkovsky, Tetrahedron Lett. 1998, 39, 139-142.

[35] a) P. A. Roethle, P. T. Hernandez, D. Trauner, Org. Lett. 2006, 8 , 5901-5904; b) see also P. Huang, V. H. Rawal, Org. Lett., 2006, 8 , 573-545.

[36] a) B. Tang, C. D. Bray, G. Pattenden, Tetrahedron Lett. 2006, 47, 6401-6404; b) B. Tang, C. D. Bray, G. Pattenden, Org. Biomol. Chem 2009, 7, 4448-4457

[37] S. C. Wang, D. J. Tantillo, J. Org. Chem. 2008, 73, 1516-1523.

[38] D. Stichnoth, D.; P. Kölle, T. J. Kimbrough, E. Riedle, E.; R. de VivieRiedle, D. Trauner, D. Nat. Commun. 2014, 5, 5597.

[39] J.-H. Sheu, A. F. Ahmed, R.-T. Shiue, C.-F.Dai, Y.-H. Kuo, J. Nat. Prod 2002, 65, 1904-1908.

[40] K.-E. Lillsunde, C. Festa, H. Adel, S. De Marino, V. Lombardi, S. Tilvi, D. Nawrot, A. Zampella, L. D'Souza, M. D'Auria, P. Tammela, Mar. Drugs 2014, 12, 4045-4068.

[41] W.-H. Yen, Y.-D. Su, Y.-C. Chang, Y.-H. Chen, C.-F Dai, Z.-H. Wen, J.H. Su, P.-J. Sung, Tetrahedron Lett. 2013, 54, 2267-2270.

[42] For discussion see reference 6.

[43] A. Saitman, P. Rulliere, S. D. E. Sullivan, E. A. Theodorakis, Org. Lett. 2011, 13, 5854-5857.

[44] K. Iguchi, K. Kajiyama, H. Miyaoka, Y. Yamada, J. Org. Chem. 1996 61, 5998-6000.

[45] For a recent synthesis of sinulariadiolide see: Z. Meng, A. Fürstner J. Am. Chem. Soc. 2019, 141, 805-809.

[46] M. J. Palframan, G. Pattenden, Tetrahedron Lett. 2013, 54, 6822-6825.

[47] S.-Y. Cheng, N.-L. Shih, C.-T. Chuang, S.-F. Chiou, C.-N. Yang, S.-K Wang, C.-Y. Duh, Bioorg. Med. Chem. Lett. 2014, 24, 1562-1564.

[48] Y. Li, G. Pattenden, Tetrahedron 2011, 67, 10045-10052.

[49] W.-H. Yen, L.-C. Hu, J.-H. Su, M.-C. Lu, W.-H. Twan, S.-Y. Yang, Y.-C Kuo, C.-F. Weng, C.-H. Lee, Y.-H. Kuo, and P.-J. Sung, Molecules 2012, 17, 14058-14066.

[50] Y.-J. Tseng, A. F. Ahmed, C.-F. Dai, M. Y. Chiang and J.-H. Sheu, Org Lett., 2005, 7, 3813-3816.

[51] M. M. Bandurraga, W. Fenical, S. F. Donovan, J. Clardy, J. Am. Chem Soc. 1982, 104, 6463-6465.

[52] S. A. Look, M. T. Burch, W. Fenical, Z. Qi-Tai, J. Clardy, J. Org. Chem. 1985, 50, 5741-5746.

[53] a) A. D. Rodriguez, J.-G. Shi, J. Org. Chem. 1998, 63, 420-421; b) A. D. Rodriguez, J.-G. Shi, S. D. Huang, J. Org. Chem., 1998, 63, 44254432

[54] a) Z. Yang, Y. Li, G. Pattenden, Tetrahedron 2010, 66, 6546-6549; b) Y. Li, G. Pattenden, Tetrahedron Lett. 2011, 52, 3315-3319.

[55] H. Weinstabl, T. Gaich, J. Mulzer, Org. Lett. 2012, 14, 2834-2837.

[56] For a full discussion see reference 6

[57] T. J. Kimbrough, P. A. Roethle, P. Mayer, D. Trauner, Angew. Chem. Int. Ed. 2010, 49, 2619-2621.

[58] a) N. Toelle, H. Weinstabl, T. Gaich, J. Mulzer, Angew. Chem. Int. Ed. 2014, 53, 3859-3862; b) T. Gaich, H. Weinstabl, J. Mulzer, Synlett, 2009, 1357-1366; c) J. D. White, S. J. Jana, J. Org. Chem. 2014, 79 700-710; d) J. D. White, S. J. Jana, Org. Lett. 2009, 11, 1433-1436; e) S. J. Stevens, A. Bérubé, J. L. Wood, Tetrahedron 2011, 67, 6479-6481.

[59] C. D. Bray, G. Pattenden, Tetrahedron Lett. 2006, 47, 3937-3939

[60] B. Tang, R. S. Paton, Org. Lett. 2019, 21, 1243-1247. 
Entry for the Table of Contents (Please choose one layout)

\section{MINIREVIEW}

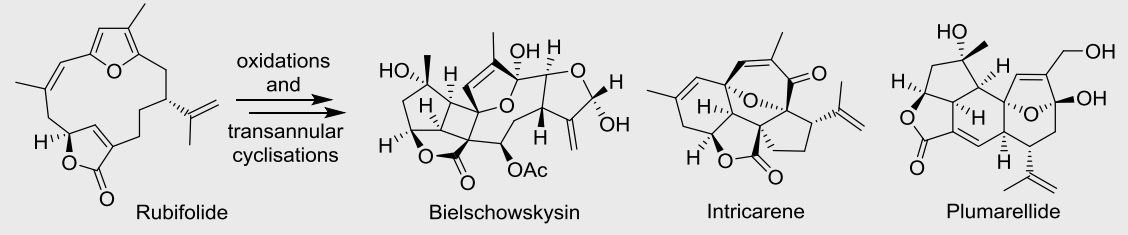

Macrocyclic furanobutenolide-based cembranoids are precursors to a wide variety of complex ring-fused diterpene structures in corals, implicating a wide variety of oxidation and transannlar cyclisations. This article gives a personal perspective on the speculations that underpin these biosynthetic interrelationships and summarises biomimetic synthesis studies designed to probe these speculations.
Biomimetic synthesis • Natural products •

Matthew J. Palframan ${ }^{[a]}$ and Gerald Pattenden*

Page No. - Page No.

Biosynthetic interrelationships within polycyclic cembranoids isolated from corals. Conjecture, biomimetic synthesis and reality. 